\title{
ORIGINAL
}

Hans Amstutz • Michael Vormwald $\mathbb{D}$

\section{Elastic spherical inhomogeneity in an infinite elastic solid: an exact analysis by an engineering treatment of the problem based on the corresponding cavity solution}

Received: 3 August 2020 / Accepted: 5 November 2020 / Published online: 11 January 2021

(C) The Author(s) 2021

\begin{abstract}
In the present work, solutions are recapitulated according to the theory of elasticity for the deformations of an adhesive spherical inhomogeneity in an infinite matrix under remote uniform axial and axialsymmetrical radial tension. Stress fields in the inhomogeneity and at the interface in the matrix are provided, too. It is shown that the sphere is deformed to a spheroid under any of the loading cases considered. Due to the axial-symmetric setup of the problem, the deformation is fully described by the two displacement values at line segments on the principal axes of the spheroid. The displacements depend on the applied remote load and on two traction fields at the inhomogeneity-matrix interface. For any combination of inhomogeneity and matrix stiffness, the condition of compatibility of deformations yields a system of two linear equations with the two magnitudes of the tractions as unknowns. Thus, the problem is reduced to a formulation for solving a twofold statically indetermined structure. The system is solved and the exact solution of the general spherical inhomogeneity problem with differing stiffness in terms of Young's moduli and Poisson's ratios of inclusion and matrix is presented.
\end{abstract}

Keywords Spherical inhomogeneity $\cdot$ Elastic inhomogeneity $\cdot$ Stress analysis $\cdot$ Strain analysis

\section{List of symbols}

$\begin{array}{ll}R & \text { Radius of the sphere } \\ \mathrm{A}, \mathrm{A}^{\prime} & \text { Pole, meridian of the sphere } \\ \mathrm{B} & \text { Equator of the sphere } \\ \mathrm{C} & \text { Surface of the sphere } \\ r, z & \text { Coordinates of an axis-symmetrical system } r-z \\ r_{\mathrm{c}}, z_{\mathrm{c}} & \text { Coordinates of a point on the surface of the sphere } \\ t_{z}, t_{r} & \text { Stress vector acting on the surface of a cut } \\ \varphi & \text { Horizontal angle in circumferential direction } \\ \theta & \text { Vertical angle } \\ \sigma_{r}, \sigma_{z}, \sigma_{\varphi} & \text { Stress components in } r-, z-, \varphi \text {-direction } \\ \tau_{r z} & \text { Shear stress component } \\ \sigma_{\mathrm{n}}, \sigma_{\mathrm{t}} & \text { Stress components in normal and tangential direction of the transformed coordinates } \\ & \text { system }\end{array}$

H. Amstutz · M. Vormwald ( $\varangle$ )

Department of Civil and Environmental Sciences, Materials Mechanics Group, Technical University of Darmstadt, FranziskaBraun-Str. 3, 64287 Darmstadt, Germany

E-mail: vormwald@wm.tu-darmstadt.de

H. Amstutz

E-mail: amstutz@wm.tu-darmstadt.de 


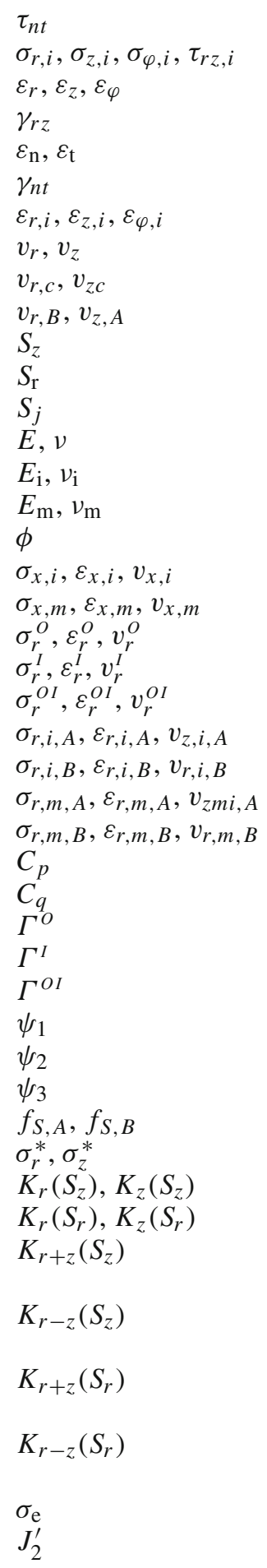

Shear stress component

Stress components in the inhomogeneity

Strain components in $r$-, $z$-, $\varphi$-direction

Shear strain component

Strain components in normal and tangential direction

Shear strain component

Strain components in the inhomogeneity

Displacement components in radial and axial direction

Displacement components of the sphere surface

Displacements at the equator (B) and the pole (A)

Load stress component in axial direction

Axial-symmetrical load stress component in radial direction

Undefined load stress

Young's modulus, Poisson's ratio of the material

Young's modulus, Poisson's ratio of the inhomogeneity material

Young's modulus, Poisson's ratio of the matrix material

Ratio $E_{\mathrm{i}} / E_{\mathrm{m}}$ of the Young's moduli

Subscript,$- i$ denoting quantities inside the inhomogeneity

Subscript ${ }_{-, m}$ denoting quantities in the matrix

Superscript ${ }^{O}$ denoting quantities of the homogeneous body

Superscript ${ }^{I}$ denoting quantities of the body with cave

Superscript ${ }^{O I}$ denoting quantities of the superposed system

Subscript $-, i, A$ denoting quantities in the inhomogeneity at the pole (A)

Subscript $-, i, B$ denoting quantities in the inhomogeneity at the equator (B)

Subscript $-, m, A$ denoting quantities on the interface of the matrix at the pole (A)

Subscript $-, i, B$ denoting quantities on the interface of the matrix at the equator (B)

$=3 / 2\left(1-v_{m}\right)\left(9+5 v_{m}\right) /\left(7-5 v_{m}\right)$ Stress function

$=-3 / 2\left(1-v_{m}\right)\left(1+5 v_{m}\right) /\left(7-5 v_{m}\right)$ Stress function

Displacement variable of the homogeneous body

Displacement variable of the body with cave

Displacement variable of the superposed system $\Gamma^{O}-\Gamma^{I}$

$=-1+\phi \cdot\left(1-C_{p}\right)$

$=+v_{i}-\phi \cdot\left(v_{m}+C_{q}\right)$

$=-1+v_{i}+\phi \cdot\left(1-v_{m}-C_{p}-C_{q}\right)$

Load specific factors

Inner stress components between inhomogeneity and matrix

Inhomogeneity stress factors resulting from axial load $S_{z}$

Inhomogeneity stress factors resulting from radial load $S_{r}$

Specific inhomogeneity stress factor $\left[K_{r}\left(S_{z}\right)+K_{z}\left(S_{z}\right)\right] / 2$ of the transformed coordinates system, axial load $S_{z}$

Specific inhomogeneity stress factor $\left[K_{r}\left(S_{z}\right)-K_{z}\left(S_{z}\right)\right] / 2$ of the transformed coordinates system, axial load $S_{z}$

Specific inhomogeneity stress factors $\left[K_{r}\left(S_{r}\right)+K_{z}\left(S_{r}\right)\right] / 2$ of the transformed coordinates system, radial load $S_{z}$

Specific inhomogeneity stress factors $\left[K_{r}\left(S_{r}\right)-+K_{z}\left(S_{r}\right)\right] / 2$ of the transformed coordinates system, radial load $S_{z}$

Equivalent stress after the maximum shear strain energy criterion

Second invariant of the stress deviator

\section{Introduction}

Inhomogeneities as inclusions or discontinuities interact with the surrounding structure generating inner restraints which affect a disturbance of the stress strain field with local stress concentrations. A first step in avoiding failure of a component consists in determining the stresses by an analysis of the problem. Generally, the analysis of non-homogeneous stress-strain fields requires the continuum-mechanical solution in the 
frame of the theory of elasticity. Irrespective of the numerical option, such as the finite element method (FEM), the analytical treatment of mathematically accessible systems is favored as a closed form exact solution given by a adequate compact formula opens insight in the mechanical connections. Such solutions are particularly suited for the takeover in handbooks and practical applications.

A first solution of a spherical inhomogeneity in an infinite solid has been achieved by Goodier [1] using harmonic functions which satisfy the equations of equilibrium and kinematics. However, the analysis is performed only for the special cases of a cavity and a rigid inhomogeneity. Edwards [2] succeeded in the analysis of an prolate spheroidal cave and inhomogeneity considering various stiffness properties in inhomogeneity and matrix. The general equations of equilibrium and kinematics are solved by a three-function approach with harmonic functions after Papcovich-Neuber in the formulation of Sadowsky and Sternberg [3] for a total of five load cases. Edwards first time drew attention to the remarkable fact that under a uniform outer load stress field the stress and strain state within the spheroidal inhomogeneity is also uniform. In his famous work Eshelby [4] showed that for an ellipsoidal inhomogeneity subject to a constant eigenstrain the total strain field within the inhomogeneity is also constant and confirmed Edward's result. It depends on the Eshelby-tensor of rank 4 with known components for an isotropic inclusion and matrix. The remarkable result of homogeneity of the stress and strain states within the inhomogeneity correlates with the deformation of the interface due to a homogeneous traction; in both inhomogeneity and matrix, the sphere is deformed to a spheroid. The traction is self-adjusting, so that in particular compatibility at the interface is fulfilled, as described in $[5,6]$ for the two-dimensional example of the circular and elliptical inhomogeneity. This correlation is also valid for spherical and ellipsoidal inhomogeneities.

All the cited solutions are derived applying complicated analytical methods which result in difficult and extended solutions. Unfortunately, their realization is commonly accompanied by a numerical evaluation, uncomfortable for application in practice. This may be the reason why inclusion and inhomogeneity problems are widely left unconsidered in handbooks of elasticity as in Roark's Formulas [7]. Peterson's Handbook of Stress Concentration Factors [8] and other catalogues [9-11] offer merely solutions for specific stiffness relations and loading situations.

The work presented here demonstrates that an exact solution of an infinite solid with a spherical inhomogeneity with mismatching elastic constants can be achieved by an engineering procedure based on the notch stresses of the corresponding cavity system and leading to a sufficiently condensed formula. Exploiting the known homogeneity of the stress-strain states within the inhomogeneity and the resulting deformation characteristics, it is concluded that there do exist contact stresses between matrix and inhomogeneity depending on the Young's moduli and Poisson's ratios of matrix and inhomogeneity. The task is to formulate an equation system of the unknown stress components which satisfies the condition of compatible deformations of the interface between matrix and inhomogeneity. The method already used in $[5,6]$ for a circular and an elliptical inhomogeneity in plates of plane stress state is here extended to the three-dimensional problem of a spherical inhomogeneity. The sought stress components in the interface follow from a superposition of the two special cases

(a) the solid with a (pseudo-)inhomogeneity of equal stiffness $E_{i}=E_{m}$ and $v_{i}=v_{m}$ and

(b) the solid with a fictitious inhomogeneity of vanishing stiffness $E_{i}=0$ and $v_{i}=0$

both under a uniform axial and an axial-symmetric radial loading.

For material strength aspects, the interest is focused on the stress concentrations in a component or the meso-structure, given by handy formulae or diagrams of the relevant stresses. In the case of inclusion and inhomogeneity, problems these are regularly found close to the interface between inhomogeneity and matrix. Therefore, the analysis presented here is for the most restricted to the determination of the stress components inside the inhomogeneity and at the interface of the matrix. Not before the very end of the paper an example is shown that the entire stress fields in the matrix can be specified having merely the remote homogeneous loading stresses and the stresses inside the inhomogeneity at hand, the latter calculated with the formulas provided here.

\section{Infinite body with a perfectly bonded spherical inhomogeneity of equal stiffness under remote uniform axial load $S_{z}$ and axial-symmetrical radial load $S_{\mathbf{r}}$}

The analysis of the axial-symmetrical problem is formulated using a cylindrical coordinate system with the coordinate $r$ for the radial and $z$ for the axial dimension, Fig. 1 . 


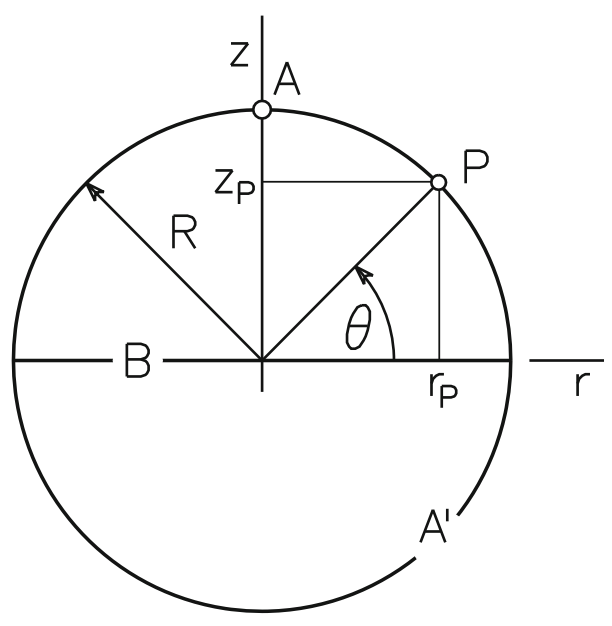

(a)

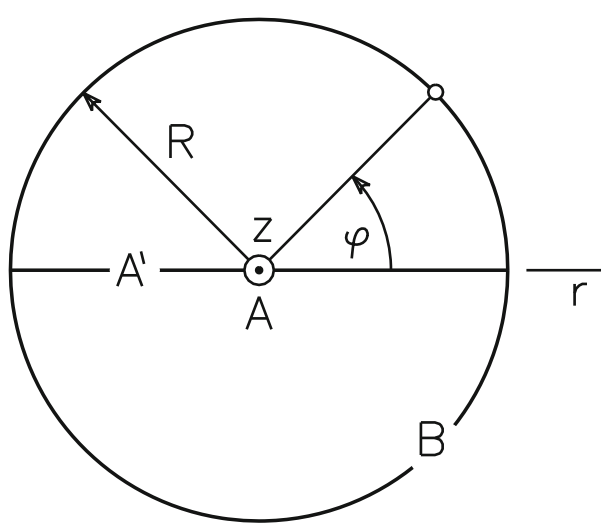

(b)

Fig. 1 Axissymmetrical coordinate system: a a $r-z$-plane, $\mathbf{b}$ the equatorial plane $z=0$

The surface of a spherical inhomogeneity can be described by the meridians $A^{\prime}$ in Fig. 2 with the pole (A) and by the equator (B).

The three-dimensional stress and strain states are determined by the stress components $\sigma_{r}, \sigma_{z}, \sigma_{\varphi}$, and the strain components $\varepsilon_{r}, \varepsilon_{z}, \varepsilon_{\varphi}$, Fig. 3 .

\subsection{Axial loading stress $S_{z}$}

Now consider an infinite axial-symmetrical body with a perfectly bonded spherical inhomogeneity with matching material parameters $E_{\mathrm{i}}=E_{\mathrm{m}}$ and $v_{\mathrm{i}}=v_{\mathrm{m}}$ loaded by a uniform remote stress $S_{z}$, Fig. 4 . This trivial limit case of the general inhomogeneity problem is identical to the homogeneous structure, subsequently marked by the superscript ${ }^{O}$. The load $S_{z}$ causes a homogeneous stress field in inhomogeneity and surrounding matrix

$$
\begin{aligned}
& \sigma_{z}^{o}=S_{z} \\
& \sigma_{r}^{o}=\sigma_{\varphi}^{o}=\tau_{z r}^{o}=0
\end{aligned}
$$

connected with a homogeneous strain field

$$
\begin{aligned}
& \varepsilon_{z}^{O}=\frac{S_{z}}{E_{\mathrm{m}}} \\
& \varepsilon_{r}^{O}=\varepsilon_{\varphi}^{O}=-v_{\mathrm{m}} \frac{S_{z}}{E_{\mathrm{m}}}
\end{aligned}
$$




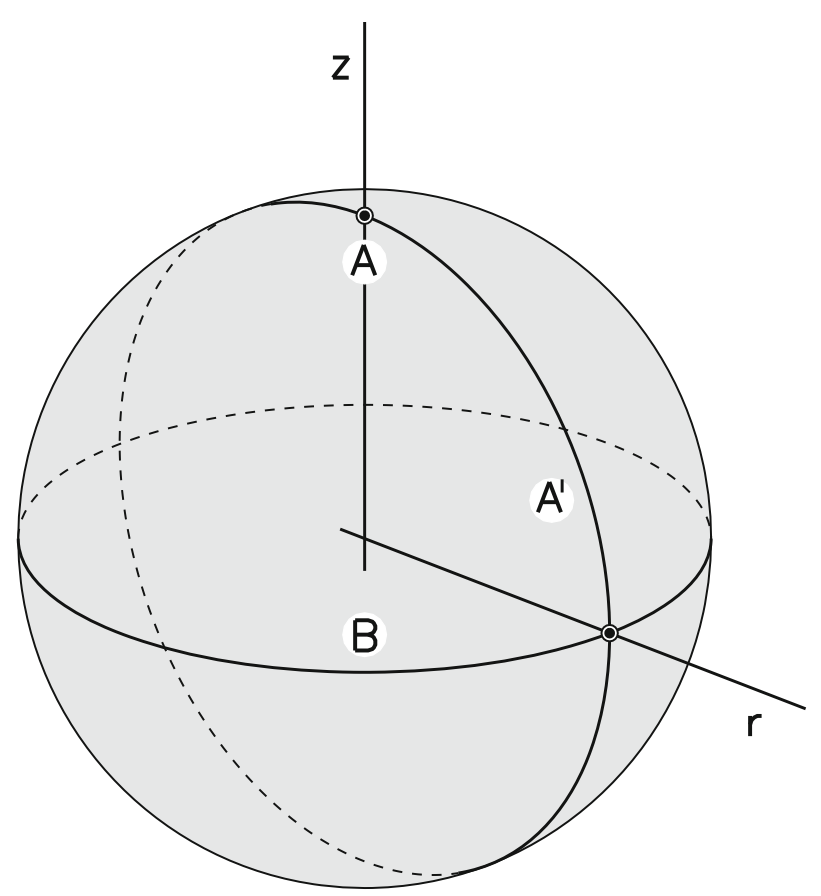

Fig. 2 Axonometric projection of a sphere with meridian $\mathrm{A}^{\prime}$, pole A and equator B
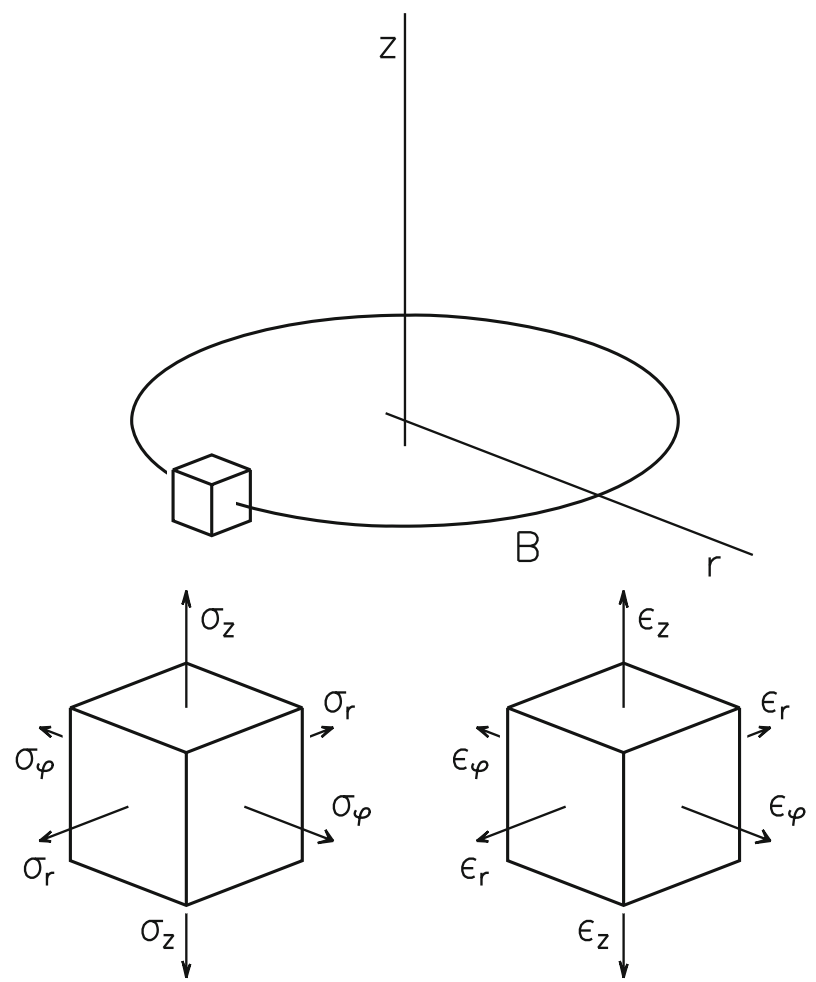

Fig. 33 D-element on equator B with the stress components $\sigma_{r}, \sigma_{z}, \sigma_{\varphi}$ and the strain components $\varepsilon_{r}, \varepsilon_{z}, \varepsilon_{\varphi}$ 


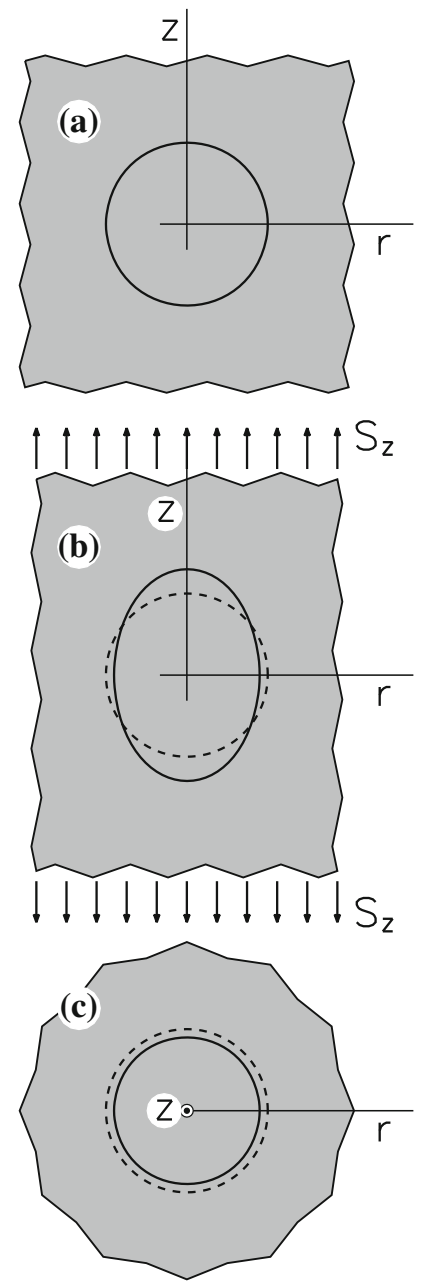

Fig. 4 Homogeneous body with a spherical region unloaded (a), loaded and deformed by an axial stress $S_{z}$ shown in a $r-z$-plane (b) and the equatorial plane $z=0(\mathbf{c})$

leading after integration to the displacements of the sphere surface $C$

$$
\begin{aligned}
& v_{z, c}^{o}\left(S_{z}\right)=\frac{z_{c}}{E_{\mathrm{m}}} S_{z} \\
& v_{r, c}^{o}\left(S_{z}\right)=-\frac{v_{\mathrm{m}} r_{c}}{E_{\mathrm{m}}} S_{z} .
\end{aligned}
$$

Displacements proportional to the radial and axial coordinates as given in Eqs. (5) and (6), Fig. 5, deform the original sphere to an ellipsoid, to say a shape of geometrical affinity of the original sphere. The correspondence of uniform strain distribution and ellipsoidal deformation becomes obvious.

With respect to the ellipsoidal distortion, the displacements of the inhomogeneity surface can be defined by the displacement values at the pole (A) and the equator (B)

$$
\begin{aligned}
& v_{z, A}^{o}\left(S_{z}\right)=\frac{R}{E_{\mathrm{m}}} S_{z}=v_{z, i, A}^{o}=v_{z, m, A}^{o} \\
& v_{r, B}^{O}\left(S_{z}\right)=-\frac{v_{\mathrm{m}} R}{E_{\mathrm{m}}} S_{z}=v_{r, i, B}^{O}=v_{r, m, B}^{O}
\end{aligned}
$$




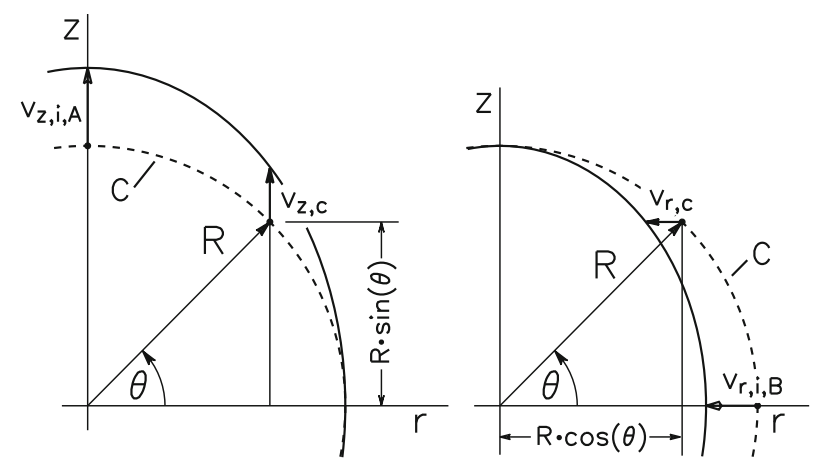

Fig. 5 Homogeneous body with a spherical region under axial loading $S_{z}$-affine displacements of the interface in the $r-z$-plane

\subsection{Axial-symmetrical radial loading stress $S_{r}$}

The axial-symmetrical radial tension load $S_{r}$ causes stresses

$$
\begin{aligned}
& \sigma_{r}^{o}=\sigma_{\varphi}^{o}=S_{r} \\
& \sigma_{z}^{o}=\tau_{r z}^{o}=0
\end{aligned}
$$

and the homogeneous strains

$$
\begin{aligned}
& \varepsilon_{r}^{o}=\varepsilon_{\varphi}^{O}=\frac{1-v_{\mathrm{m}}}{E_{\mathrm{m}}} S_{r} \\
& \varepsilon_{z}^{o}=-\frac{2 v_{\mathrm{m}}}{E_{\mathrm{m}}} S_{r} .
\end{aligned}
$$

Integration of the strains generates displacements of the sphere at $C$, Fig. 6, as describing again ellipsoidal deformations, Fig. 7, which are adequately defined by

$$
\begin{gathered}
v_{z, c}^{o}\left(S_{r}\right)=-\frac{2 v_{\mathrm{m}} z_{c}}{E_{\mathrm{m}}} S_{r} \\
v_{r, c}^{o}\left(S_{r}\right)=\frac{\left(1-v_{\mathrm{m}}\right) r_{c}}{E_{\mathrm{m}}} S_{r} \\
v_{z, A}^{O}\left(S_{r}\right)=-\frac{2 v_{\mathrm{m}} R}{E_{\mathrm{m}}} S_{r}=v_{z, i, A}^{o}=v_{z, m, A}^{O} \\
v_{r, B}^{O}\left(S_{r}\right)=\frac{\left(1-v_{\mathrm{m}}\right) R}{E_{\mathrm{m}}} S_{r}=v_{r, i, B}^{O}=v_{r, m, B}^{O}
\end{gathered}
$$

\section{Infinite body with a spherical cave under remote uniform axial load $S_{z}$ and axial-symmetric radial $\operatorname{load} S_{r}$}

The body with a spherical cave can be considered here as being a second limit case of the inhomogeneity problem. This case, in the following denoted by the superscript ${ }^{I}$, is characterized by a complete loss of stiffness with Young's modulus $E_{\mathrm{i}}=0$, leading to a zero stress within the fictitious inhomogeneity region,

$$
\sigma_{z, i}^{I}=\sigma_{r, i}^{I}=\tau_{z r, i}^{I}=0 .
$$

Although stresses have vanished in the inhomogeneity region, a strain field may be defined. The constant strain components can be obtained from the matrix strains at the cave surface, Fig. 8. At the pole (A) compatibility demands 

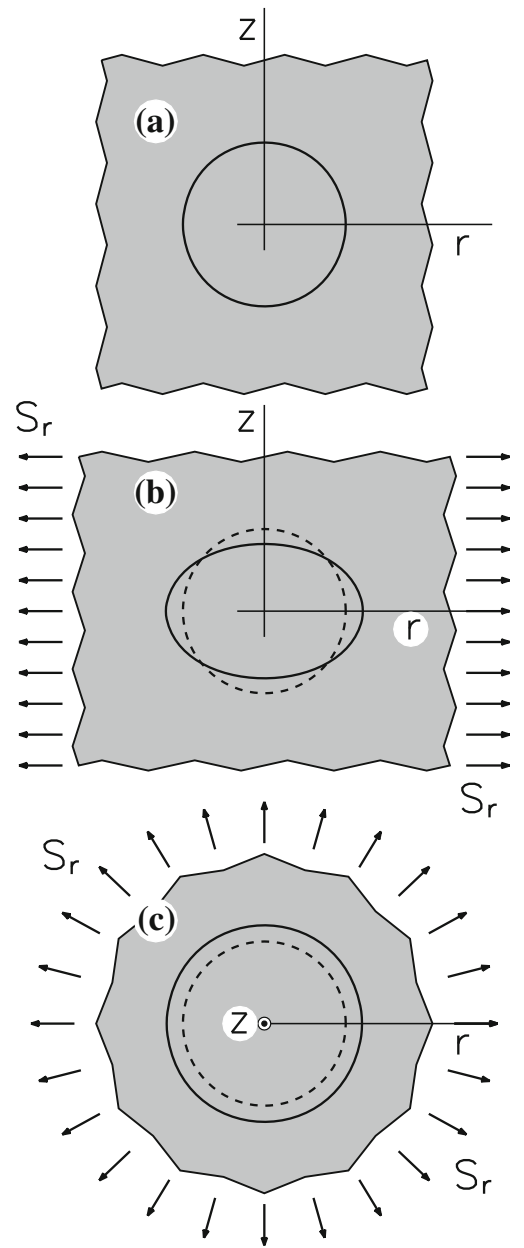

Fig. 6 Homogeneous body with a spherical region unloaded (a), loaded and deformed by an radial stress $S_{r}$ shown in a $r-z$-plane (b) and the equatorial plane $z=0$ (c)

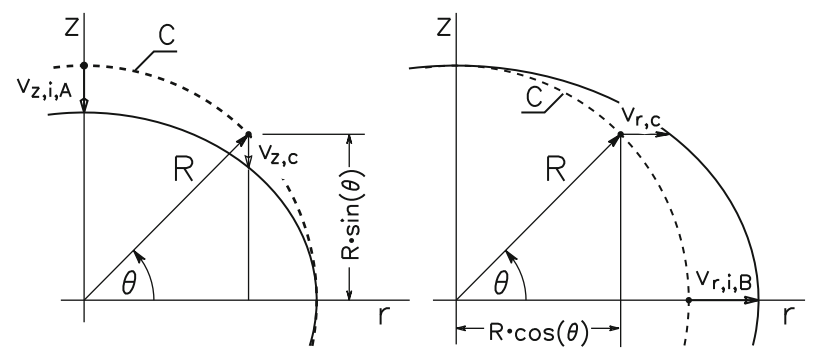

Fig. 7 Homogeneous body with a spherical region under radial loading $S_{r}$ affine displacements of the interface in the $r-z$-plane

$$
\varepsilon_{r, i, A}^{I}=\varepsilon_{r, m, A}^{I}
$$

and at the equator (B)

$$
\begin{aligned}
\varepsilon_{z, i, B}^{I} & =\varepsilon_{z, m, B}^{I} \\
\varepsilon_{\varphi, i, B}^{I} & =\varepsilon_{\varphi, m, B}^{I}
\end{aligned}
$$




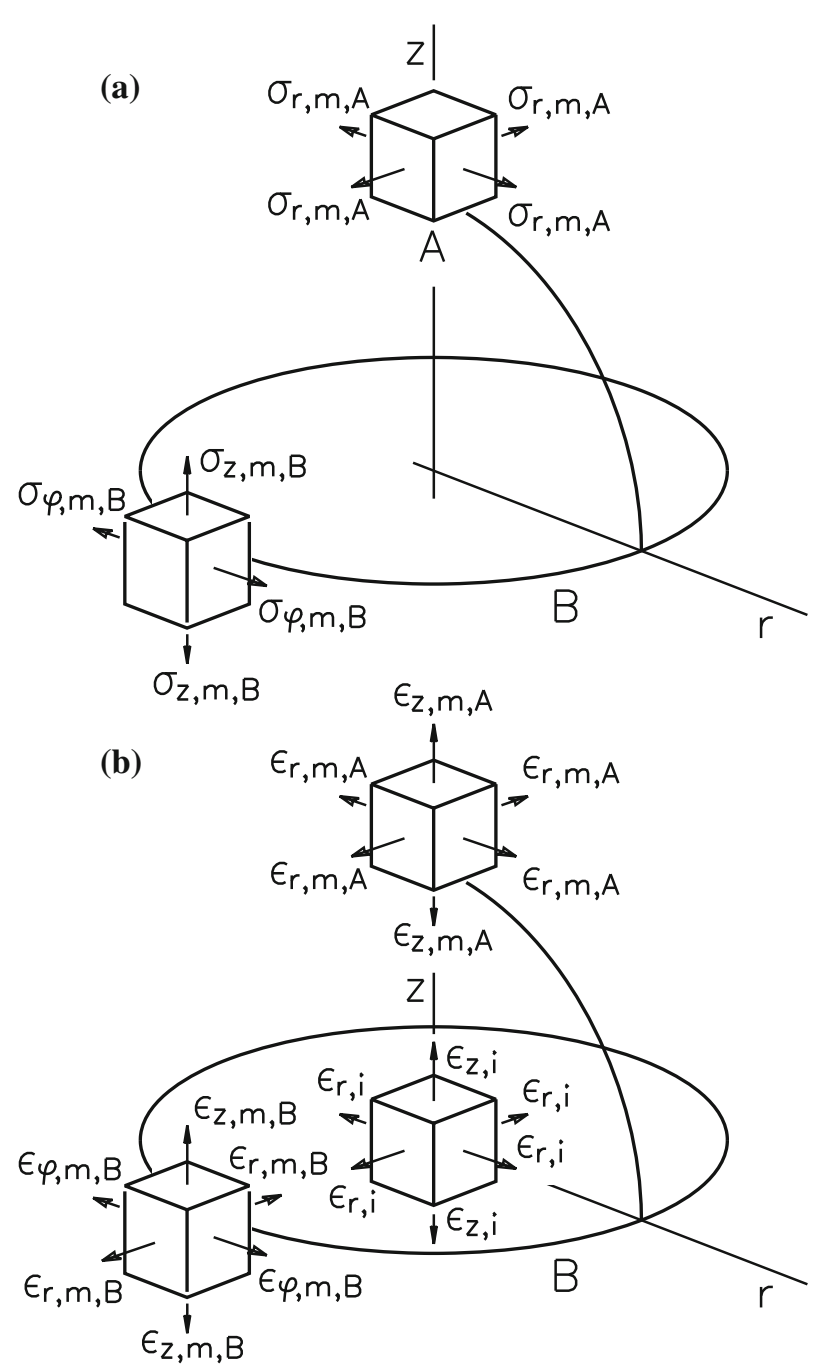

Fig. 8 Body with a spherical cave under axial loading $S_{z}$-stress states at the cave surface (a) and strain states at the cave surface and fictitious strains inside the cave (b)

where $\varepsilon_{z, i, B}^{I}, \varepsilon_{\varphi, i, B}^{I}$ are the fictitious strain components of the inhomogeneity and $\varepsilon_{z, m, B}^{I}, \varepsilon_{\varphi, m, B}^{I}$ the strains of the matrix at (B) and the radial components $\varepsilon_{r, i, A}^{I}, \varepsilon_{r, m, A}^{I}$ at (A) all in tangential direction as shown in Fig. 8. The strain components $\varepsilon_{z, m, B}^{I}, \varepsilon_{\varphi, m, B}^{I}$ and $\varepsilon_{\varphi, m, A}^{I}$ are correlated with the tangential stress components by

$$
\begin{aligned}
& \varepsilon_{z, m, B}^{I}=\frac{1}{E_{\mathrm{m}}}\left(\sigma_{z, m, B}^{I}-v_{\mathrm{m}} \sigma_{\varphi, m, B}^{I}\right)=\varepsilon_{z, i, B}^{I} \\
& \varepsilon_{\varphi, m, B}^{I}=\frac{1}{E_{\mathrm{m}}}\left(\sigma_{\varphi, m, B}^{I}-v_{\mathrm{m}} \sigma_{z, m, B}^{I}\right)=\varepsilon_{\varphi, i, B}^{I} \\
& \varepsilon_{r, m, A}^{I}=\frac{1}{E_{\mathrm{m}}} \sigma_{r, m, A}^{I}\left(1-v_{\mathrm{m}}\right)=\varepsilon_{r, i, A}^{I}
\end{aligned}
$$

and assuming uniform strain distribution inside the inhomogeneity region

$$
\begin{aligned}
\varepsilon_{z, i}^{I} & =\varepsilon_{z, i, B}^{I} \\
\varepsilon_{\varphi, i}^{I} & =\varepsilon_{\varphi, i, B}^{I} \\
\varepsilon_{r, i}^{I} & =\varepsilon_{r, i, A}^{I}
\end{aligned}
$$

where $\varepsilon_{r, i}^{I}$ has to be identical with $\varepsilon_{\varphi, i}^{I}$. 
3.1 Axial loading stress $S_{z}$

The stress $\sigma_{z, m, B}^{I}, \sigma_{\varphi, m, B}^{I}$ and $\sigma_{r, m, A}^{I}$ can be taken from the solution of Neuber [12] in 1937 and other publications $[1,8]$. For axial loading $S_{z}$, the stress components at the pole (A) read

$$
\sigma_{r, m, A}^{I}=-\frac{3\left(1+5 v_{\mathrm{m}}\right)}{2\left(7-5 v_{\mathrm{m}}\right)} S_{z}
$$

and at the equator $(B)$

$$
\begin{aligned}
\sigma_{z, m, B}^{I} & =\frac{3\left(9-5 v_{\mathrm{m}}\right)}{2\left(7-5 v_{\mathrm{m}}\right)} S_{z} \\
\sigma_{\varphi, m, B}^{I} & =\frac{3\left(5 v_{\mathrm{m}}-1\right)}{2\left(7-5 v_{\mathrm{m}}\right)} S_{z}
\end{aligned}
$$

Introducing the composed stress factors

$$
\begin{aligned}
& \sigma_{z, m, B}^{I}-v_{\mathrm{m}} \sigma_{\varphi, m, B}^{I}=\frac{3}{2}\left(1-v_{\mathrm{m}}\right)\left[\frac{9+5 v_{\mathrm{m}}}{7-5 v_{\mathrm{m}}}\right] S_{z}=C_{p} S_{z} \\
& \sigma_{\varphi, m, B}^{I}-v_{\mathrm{m}} \sigma_{z, m, B}^{I}=-\frac{3}{2}\left(1-v_{\mathrm{m}}\right)\left[\frac{1+5 v_{\mathrm{m}}}{7-5 v_{\mathrm{m}}}\right] S_{z}=C_{q} S_{z}
\end{aligned}
$$

there follows from Eqs. (21) to (23) for the strain components

$$
\begin{aligned}
\varepsilon_{z, m, B}^{I} & =\varepsilon_{z, i, B}^{I}=\varepsilon_{z, i}^{I} \\
& =\frac{1}{E_{\mathrm{m}}}\left(\sigma_{z, m, B}^{I}-v_{\mathrm{m}} \sigma_{\varphi, m, B}^{I}\right)=\frac{C_{p}}{E} S_{z} \\
\varepsilon_{\varphi, m, B}^{I} & =\varepsilon_{\varphi, i, B}^{I}=\varepsilon_{\varphi, i}^{I} \\
& =\frac{1}{E_{\mathrm{m}}}\left(\sigma_{\varphi, m, B}^{I}-v_{\mathrm{m}} \sigma_{z, m, B}^{I}\right)=\frac{C_{q}}{E} S_{z} \\
\varepsilon_{r, m, A}^{I} & =\varepsilon_{r, i, A}^{I}=\varepsilon_{r, i}^{I} \\
& =\frac{1}{E_{\mathrm{m}}}\left(1-v_{\mathrm{m}}\right) \sigma_{r, m, A}^{I}=\frac{C_{q}}{E} S_{z}
\end{aligned}
$$

The identity of Eqs. (33) and (34) together with Eqs. (27) and (31) demonstrates the constancy of the strain field inside the inhomogeneity. This homogeneous strain distribution causes again an ellipsoidal deformation, Fig. 9, which can be described after integration of Eqs. (32) and (33) by the displacements at the surface $c$ by

$$
v_{z, m, c}^{I}\left(S_{z}\right)=\frac{C_{p} z_{c}}{E_{\mathrm{m}}} S_{z}
$$

and

$$
v_{r, m, c}^{I}\left(S_{z}\right)=\frac{C_{q} r_{c}}{E_{\mathrm{m}}} S_{z}
$$

leading at the pole (A) and the equator (B), Fig. 10, to

$$
\begin{aligned}
& v_{z, m, A}^{I}\left(S_{z}\right)=\frac{R C_{p}}{E_{\mathrm{m}}} S_{z} \\
& v_{r, m, B}^{I}\left(S_{z}\right)=\frac{R C_{q}}{E_{\mathrm{m}}} S_{z}
\end{aligned}
$$




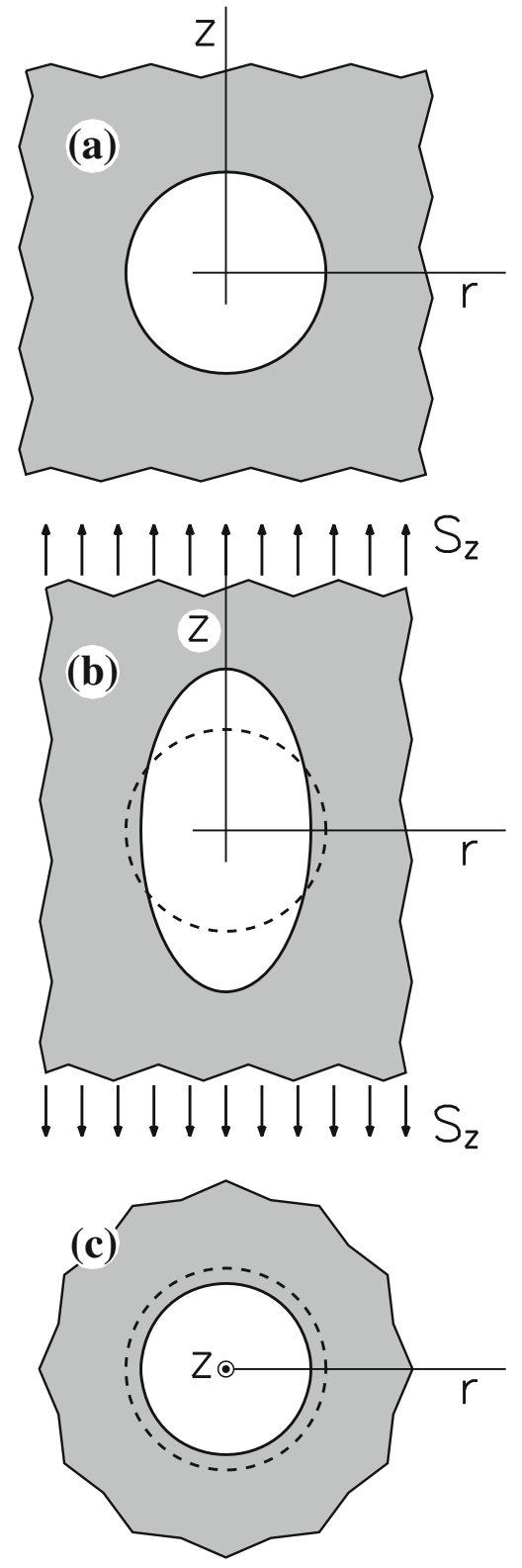

Fig. 9 Body with a spherical cave unloaded (a), loaded and deformed by an axial stress $S_{z}$ shown in a $r-z$-plane (b) and the equatorial plane $z=0$ (c)
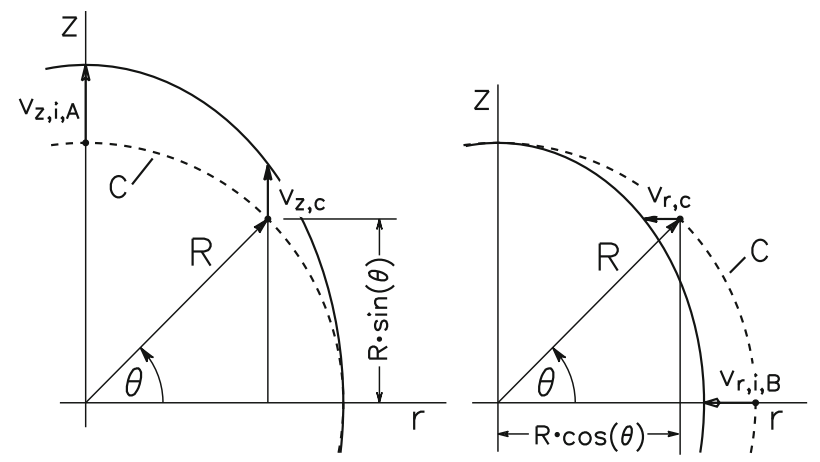

Fig. 10 Body with a spherical cave under radial loading $S_{z}$ affine displacements of the cave surface in the $r-z$-plane 
3.2 Axial-symmetrical radial loading stress $S_{r}$

The significant stresses for the axial-symmetrical radial loading case $S_{r}$ can be realized by superposition of a biaxial load case with load stresses $S_{x}=S_{y}=S_{r}$. At the pole (A) this leads to

$$
\sigma_{r, m, A}^{I}=\frac{3}{2} \cdot \frac{8}{7-5 v_{\mathrm{m}}} S_{r}
$$

At the equator (B), the following stress components arise

$$
\begin{aligned}
& \sigma_{z, m, B}^{I}=-\frac{3}{2} \cdot \frac{2}{7-5 v_{\mathrm{m}}} S_{r} \\
& \sigma_{\varphi, m, B}^{I}=\frac{3}{2} \cdot \frac{8-10 v_{\mathrm{m}}}{7-5 v_{\mathrm{m}}} S_{r}
\end{aligned}
$$

In the radial load case, the composed stress factors give

$$
\begin{aligned}
& \sigma_{z, m, B}^{I}-v_{\mathrm{m}} \sigma_{\varphi, m, B}^{I}=-\frac{3}{2}\left(1-v_{\mathrm{m}}\right)\left[\frac{2+10 v_{\mathrm{m}}}{7-5 v_{\mathrm{m}}}\right] S_{r}=2 C_{q} S_{r} \\
& \sigma_{\varphi, m, B}^{I}-v_{\mathrm{m}} \sigma_{z, m, B}^{I}=\frac{3}{2}\left(1-v_{\mathrm{m}}\right)\left[\frac{8}{7-5 v_{\mathrm{m}}}\right] S_{r}=\left(C_{p}+C_{q}\right) S_{r}
\end{aligned}
$$

The strain states at (A) and (B) are correlated with the stress states according to Eqs. (21)-(23) by

$$
\begin{aligned}
\varepsilon_{z, m, B}^{I} & =\varepsilon_{z, i, B}^{I}=\varepsilon_{z, i}^{I} \\
& =\frac{1}{E_{\mathrm{m}}}\left(\sigma_{z, m, B}^{I}-v_{\mathrm{m}} \sigma_{\varphi, m, B}^{I}\right)=\frac{2 C_{q}}{E} S_{r} \\
\varepsilon_{\varphi, m, B}^{I} & =\varepsilon_{\varphi, i, B}^{I}=\varepsilon_{\varphi, i}^{I} \\
& =\frac{1}{E_{\mathrm{m}}}\left(\sigma_{\varphi, m, B}^{I}-v_{\mathrm{m}} \sigma_{z, m, B}^{I}\right)=\frac{\left(C_{p}+C_{q}\right)}{E} S_{r} \\
\varepsilon_{r, m, A}^{I} & =\varepsilon_{r, i, A}^{I}=\varepsilon_{r, i}^{I} \\
& =\frac{1}{E_{\mathrm{m}}}\left(1-v_{\mathrm{m}}\right) \sigma_{r, m, A}^{I}=\frac{\left(C_{p}+C_{q}\right)}{E} S_{r}
\end{aligned}
$$

Integrating Eqs. (44) and (45) delivers the proportional displacements of the ellipsoidal deformation of the sphere, Fig. 11

$$
\begin{aligned}
& v_{z, m, c}^{I}\left(S_{r}\right)=\frac{2 C_{q} z_{c}}{E_{\mathrm{m}}} S_{r} \\
& v_{r, m, c}^{I}\left(S_{r}\right)=\frac{\left(C_{p}+C_{q}\right) r_{c}}{E_{\mathrm{m}}} S_{r}
\end{aligned}
$$

and at the pole (A) and the equator (B), Fig. 12

$$
\begin{aligned}
& v_{z, m, A}^{I}\left(S_{r}\right)=\frac{2 R C_{q}}{E_{\mathrm{m}}} S_{r} \\
& v_{r, m, B}^{I}\left(S_{r}\right)=\frac{R\left(C_{p}+C_{q}\right)}{E_{\mathrm{m}}} S_{r}
\end{aligned}
$$




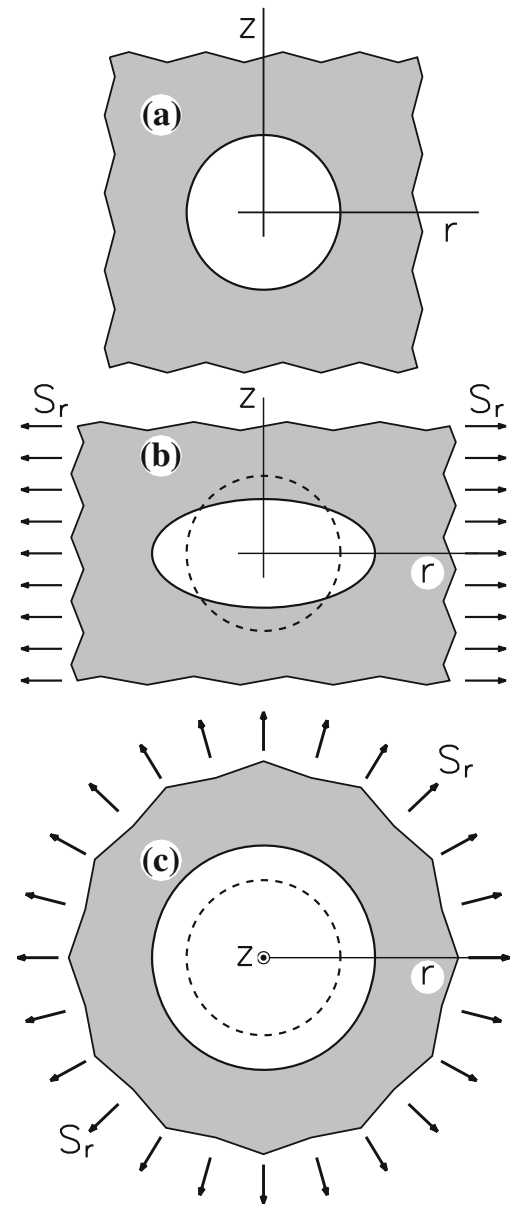

Fig. 11 Body with a spherical cave unloaded (a), loaded and deformed by a radial stress $S_{r}$ shown in a $r-z$-plane (b) and the equatorial plane $z=0$, $\mathbf{c}$ axissymmetrical coordinate system $r-z$
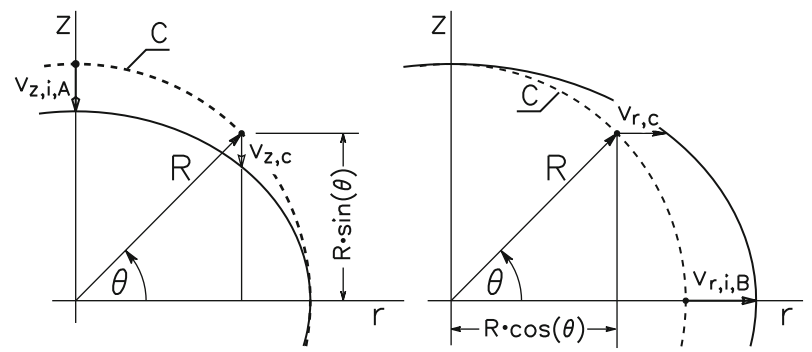

Fig. 12 Body with a spherical cave under radial loading $S_{r}$ affine displacements of the cave surface in the $r-z$-plane

\section{Load cases stress in the interface between matrix and spherical inhomogeneity}

To solve the problem of an elastic solid with an elastic inhomogeneous region of different Young's modulus and Poisson's ratio under remote loading, the method of equivalent eigenstrain may be chosen as in [13]. But in this work for the sake of mathematical simplicity, a strategy is pursued to get the solution by a superposition of the solutions of three loading cases:

(A) The solid with cave under outer loading.

(B) The solid with cave under a traction on the cave's surface. When the reaction of the traction is applied on the surface of the inhomogeneity, it is under a homogeneous state of stress, $\sigma_{z}^{*}$. Therefore, the traction is $t_{z}^{*}=\sigma_{z}^{*} \cdot n_{z}$ where $n_{z}$ is the $z$-component of the unit normal vector on the sphere's surface. 
(C) The solid with cave under another traction on the cave's surface. When the reaction of this traction is applied on the surface of the inhomogeneity, it is under a homogeneous rotationally symmetric state of stress, $\sigma_{r}^{*}$. Therefore, the traction is $t_{r}^{*}=\sigma_{r}^{*} \cdot n_{r}$ where $n_{r}$ is the $r$-component of the unit normal vector on the sphere's surface.

The relevant stresses $\sigma_{z}^{*}$ or $\sigma_{r}^{*}$ have to be determined from the displacements of all parts of the superposed system satisfying the condition of compatible deformations while considering the different Young's moduli and Poisson's ratios in matrix and inhomogeneity. The solutions for the special loading cases of the solid with cave under such tractions on the cave surface as noted in cases (B) and (C) explained in the list above can be derived in advance. This requires the superposition of the solution of the homogeneous solid and the solid with cave, both under outer loading but opposed load direction

$$
\Gamma^{O I}=\Gamma^{O}-\Gamma^{I} .
$$

As a result of the sign change in the loading of the two systems, the stress field in some distance of the interface tends to zero. The stresses in the matrix are concentrated to the neighborhood of the interface.

\subsection{Axial loading stress $S_{z}$}

The superposition according to Eq. (51) is performed here for the displacements of the inner surface at the pole (A) and the equator (B) inserting Eqs. (7), (8), (37) and (38) results in

$$
\begin{aligned}
& v_{z, m, A}^{O I}\left(S_{z}\right)=\frac{R}{E_{\mathrm{m}}} S_{z}-\frac{R C_{p}}{E_{\mathrm{m}}} S_{z}=\frac{R\left(1-C_{p}\right)}{E_{\mathrm{m}}} S_{z} \\
& v_{r, m, B}^{O I}\left(S_{z}\right)=-\frac{R v_{\mathrm{m}}}{E_{\mathrm{m}}} S_{z}-\frac{R C_{q}}{E_{\mathrm{m}}} S_{z}=-\frac{R\left(v_{\mathrm{m}}+C_{q}\right)}{E_{\mathrm{m}}} S_{z}
\end{aligned}
$$

In the following derivations of Sect. 5, the load case shown in Fig. 13c is treated as an independent load, case B in above list. The stresses $S_{z}$ appearing in Eqs. (52) and (53) are therefore renamed to $\sigma_{z}^{*}$.

$$
\begin{aligned}
& v_{z, m, A}^{O I}\left(\sigma_{z}^{*}\right)=\frac{R\left(1-C_{p}\right)}{E_{\mathrm{m}}} \sigma_{z}^{*} \\
& v_{r, m, B}^{O I}\left(\sigma_{z}^{*}\right)=-\frac{R\left(v_{\mathrm{m}}+C_{q}\right)}{E_{\mathrm{m}}} \sigma_{z}^{*} .
\end{aligned}
$$

Unnecessary to mention that the superposition according to Fig. (13) and Eq. (51) can be performed for any stress-, strain-, or displacement-field variable. The stress fields of the loading cases according to Fig. 13a, $\mathrm{b}$ are available in analytic form, see e.g., [12], and so are the fields for the load case according to Fig. 13c. Nevertheless, it is sufficient here to restrict to the two values of Eqs. (54) and (55).

\subsection{Axial-symmetrical radial loading stress $S_{r}$}

In strict analogy, see Fig. 14, the superposition of the axial-symmetrical load case $S_{r}$ after Eq. (51) produces the displacements of the interface with Eqs. (15), (16), (49) and (50) as

$$
\begin{aligned}
& v_{z, m, A}^{O I}\left(S_{r}\right)=-\frac{2 R v_{\mathrm{m}}}{E_{\mathrm{m}}} S_{r}-\frac{2 R C_{q}}{E_{\mathrm{m}}} S_{r}=-\frac{2 R\left(v_{\mathrm{m}}+C_{q}\right)}{E_{\mathrm{m}}} S_{r} \\
& v_{r, m, B}^{O I}\left(S_{r}\right)=\frac{\left(1-v_{\mathrm{m}}\right) R}{E_{\mathrm{m}}} S_{r}-\frac{R\left(C_{p}+C_{q}\right)}{E_{\mathrm{m}}} S_{r}=\frac{R\left(1-v_{\mathrm{m}}-C_{p}-C_{q}\right)}{E_{\mathrm{m}}} S_{r}
\end{aligned}
$$

and after renaming $S_{r}$ to $\sigma_{r}^{*}$

$$
\begin{aligned}
& v_{z, m, A}^{O I}\left(\sigma_{r}^{*}\right)=-\frac{2 R\left(v_{\mathrm{m}}+C_{q}\right)}{E_{\mathrm{m}}} \sigma_{r}^{*} \\
& v_{r, m, B}^{O I}\left(\sigma_{r}^{*}\right)=\frac{R\left(1-v_{\mathrm{m}}-C_{p}-C_{q}\right)}{E_{\mathrm{m}}} \sigma_{r}^{*}
\end{aligned}
$$


(a)
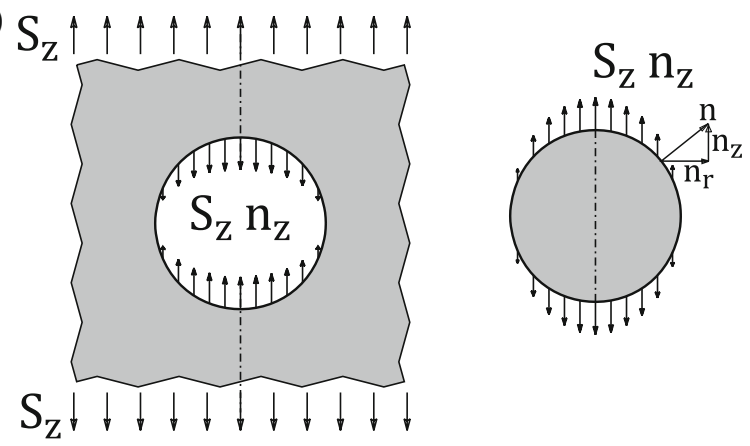

(b)
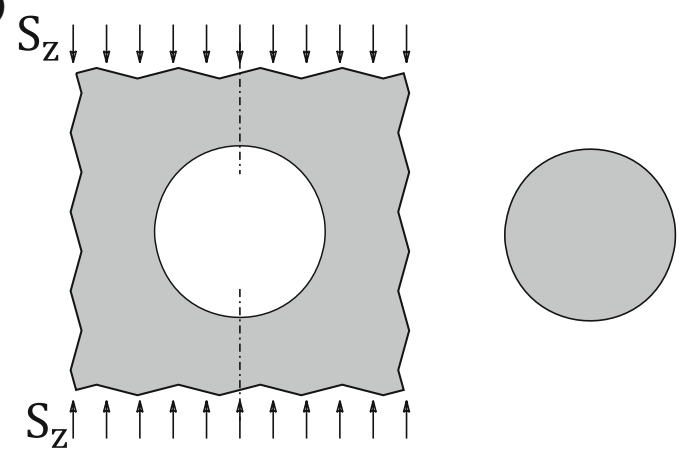

(c)
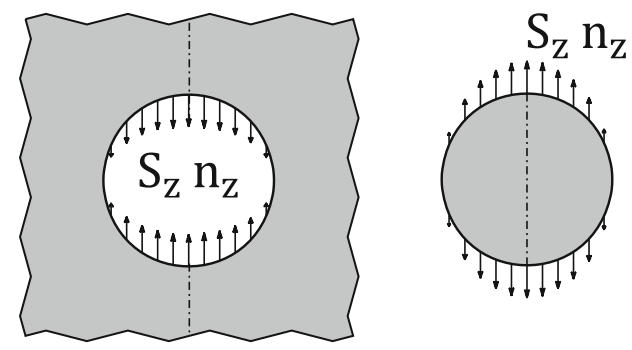

Fig. 13 Scheme to the superposition according to Eq. (51) for axial loading a homogeneous body, $\mathbf{b}$ body with cave, $\mathbf{c}$ body with cave under inner and inhomogeneity under outer stress $t_{z}=S_{z} \cdot n_{z}$

\section{Infinite solid with a spherical inhomogeneity under axial load $S_{z}$ and axial-symmetrical radial load $S_{r}$}

The correlation between deformation kinematics and stress-strain-states in an infinite solid with a spherical inhomogeneity offer an opportunity for the analysis of the general problem with non-matching stiffness of matrix and inhomogeneity. For this purpose, the system is separated fictitiously into three parts, Fig. 15,

(a) the solid with spherical cave under an outer uniform load $S_{j}$,

(b) the spherical inhomogeneity under tractions on its surface causing constant stresses $\sigma_{z}^{*}, \sigma_{r}^{*}$ inside, and

(c) the solid with spherical cave where the same tractions re-act on the cave surface.

Compatible displacements of the interface between matrix and inhomogeneity are provided by an appropriate choice of the inner stress components $\sigma_{z}^{*}$ and $\sigma_{r}^{*}$, considering the different material properties in inhomogeneity and matrix.

In respect of the general geometrical affinity in the ellipsoidal deformations, the condition of compatibility (according to the method of compatible deformations of a twofold statically indetermined system) may be 


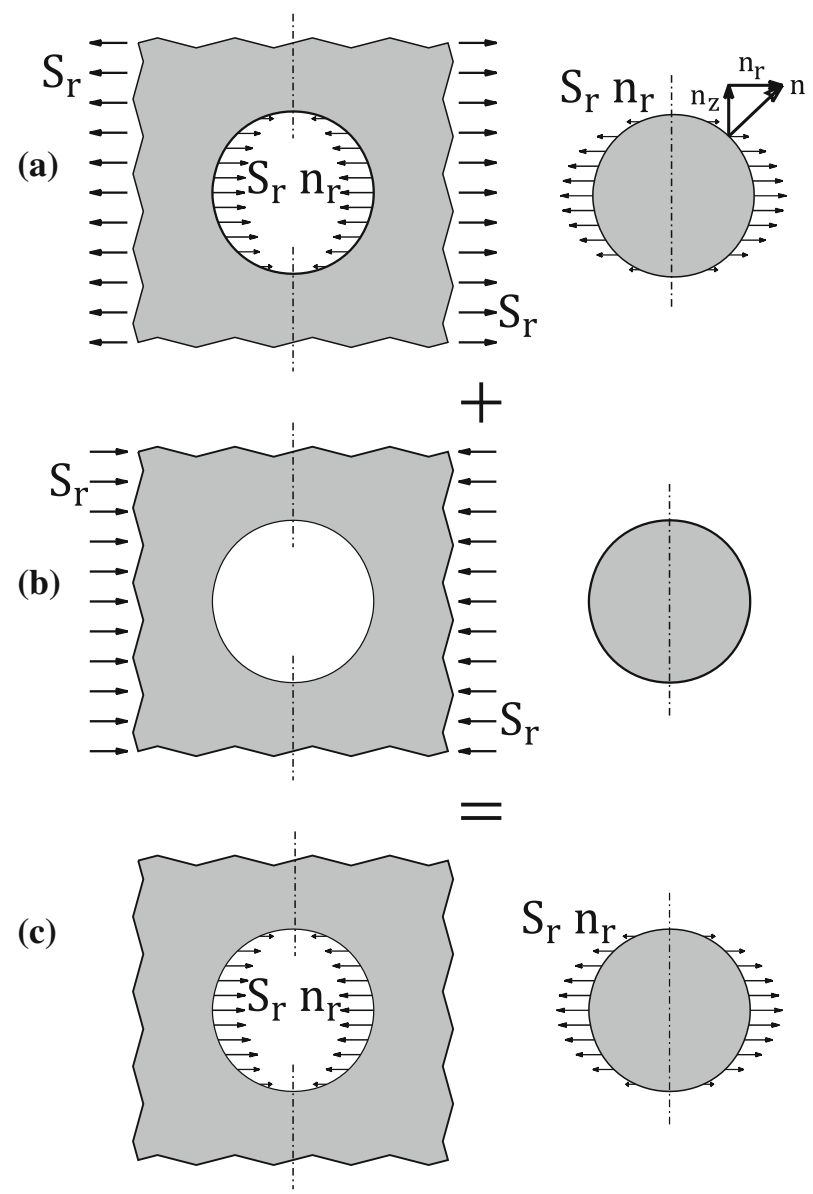

Fig. 14 Scheme to the superposition according to Eq. (51) for radial loading a homogeneous body, b body with cave, $\mathbf{c}$ body with cave under inner and inhomogeneity under outer stress $t_{r}=S_{r} \cdot n_{r}$

formulated on the basis of an equation system referring to the displacements at the pole (A) and at the equator (B), Fig. 15. In general form, the conditions are given by

$$
\begin{aligned}
& -v_{z, i, A}^{O}\left(\sigma_{z}^{*}, \sigma_{r}^{*}\right)+v_{z, m, A}^{O I}\left(\sigma_{z}^{*}, \sigma_{r}^{*}\right)+v_{z, m, A}^{I}\left(S_{j}\right)=0 \\
& -v_{r, i, B}^{O}\left(\sigma_{z}^{*}, \sigma_{r}^{*}\right)+v_{r, m, B}^{O I}\left(\sigma_{z}^{*}, \sigma_{r}^{*}\right)+v_{r, m, B}^{I}\left(S_{j}\right)=0
\end{aligned}
$$

where $v_{z, m, A}^{I}\left(S_{j}\right)$ and $v_{r, m, B}^{I}\left(S_{j}\right)$ are the displacements of the cave surface under the outer axial load $S_{z}$ or $S_{r}$. These displacements have to be compensated by the displacements of the inhomogeneity under the unknown inner stress $\sigma_{z}^{*}$ and $\sigma_{r}^{*}$ after Eqs. (7), (8), (15) and (16)

$$
\begin{aligned}
& v_{z, i, A}^{O}\left(\sigma_{z}^{*}, \sigma_{r}^{*}\right)=\frac{R}{E_{\mathrm{i}}} \sigma_{z}^{*}-\frac{2 v_{i} R}{E_{\mathrm{i}}} \sigma_{r}^{*} \\
& v_{r, i, B}^{O}\left(\sigma_{z}^{*}, \sigma_{r}^{*}\right)=-\frac{v_{i} R}{E_{\mathrm{i}}} \sigma_{z}^{*}+\frac{\left(1-v_{\mathrm{i}}\right) R}{E_{\mathrm{i}}} \sigma_{r}^{*}
\end{aligned}
$$

and the displacements of the inner stress on the cave surface of the solid regarding Eqs. (54), (55), (58) and (59)

$$
\begin{aligned}
& v_{z, m, A}^{O I}\left(\sigma_{z}^{*}, \sigma_{r}^{*}\right)=\frac{R\left(1-C_{p}\right)}{E_{\mathrm{m}}} \sigma_{z}^{*}-\frac{2 R\left(v_{\mathrm{m}}+C_{q}\right)}{E_{\mathrm{m}}} \sigma_{r}^{*} \\
& v_{r, m, B}^{O I}\left(\sigma_{z}^{*}, \sigma_{r}^{*}\right)=-\frac{R\left(v_{\mathrm{m}}+C_{q}\right)}{E_{\mathrm{m}}} \sigma_{z}^{*}+\frac{R\left(1-v_{\mathrm{m}}-C_{p}-C_{q}\right)}{E_{\mathrm{m}}} \sigma_{r}^{*}
\end{aligned}
$$



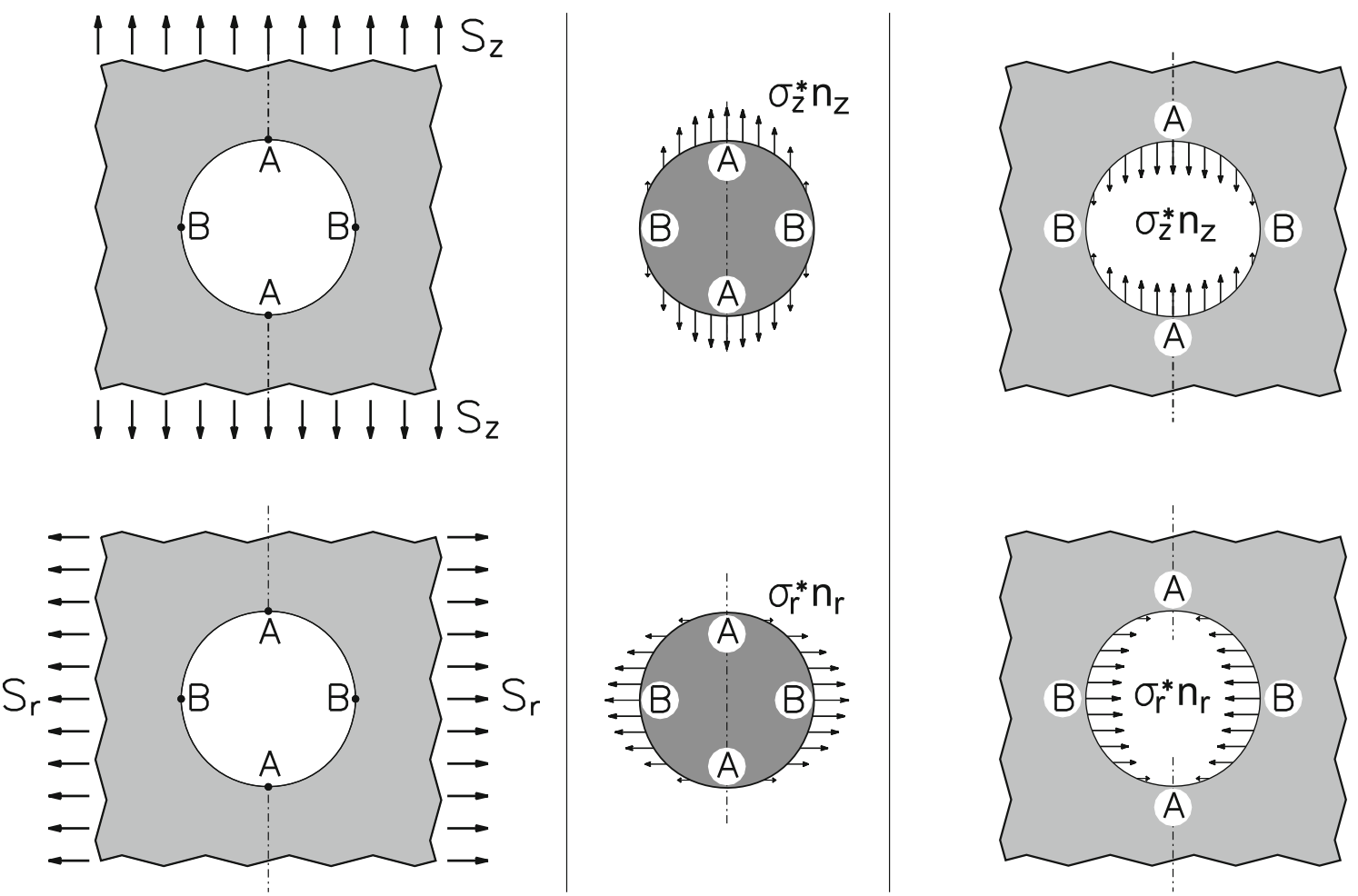

Fig. 15 Superposition scheme after Eqs. (62) and (63) to derive the inner stress $\sigma_{z}^{*}$ and $\sigma_{r}^{*}$ ensuring compatible deformations between matrix and inhomogeneity

Inserting all quantities in Eqs. (60) and (61) leads to an equation system for the determination of the two inner unknown stress components $\sigma_{z}^{*}$ and $\sigma_{r}^{*}$ in dependence of the load stress $S_{z}$ or $S_{r}$ and the material parameters $E_{\mathrm{i}}$ and $v_{\mathrm{i}}$ of the inhomogeneity and $E_{\mathrm{m}}$ and $v_{\mathrm{m}}$ of the matrix, respectively

(A)

$$
\begin{aligned}
& -\frac{R}{E_{\mathrm{i}}}\left(\sigma_{z}^{*}-2 v_{\mathrm{i}} \sigma_{r}^{*}\right)+\frac{R}{E_{\mathrm{m}}}\left[\left(1-C_{p}\right) \sigma_{z}^{*}-2\left(v_{\mathrm{m}}+C_{q}\right) \sigma_{r}^{*}\right]+\frac{R}{E_{\mathrm{m}}} f_{S, A} S_{j}=0 \\
& \quad \frac{R}{E_{\mathrm{i}}}\left[v_{\mathrm{i}} \sigma_{z}^{*}-\left(1-v_{\mathrm{i}}\right) \sigma_{r}^{*}\right]-\frac{R}{E_{\mathrm{m}}}\left[\left(v_{\mathrm{m}}+C_{q}\right) \sigma_{z}^{*}-\left(1-v_{\mathrm{m}}-C_{p}-C_{q}\right) \sigma_{r}^{*}\right]+\frac{R}{E_{\mathrm{m}}} f_{S, B} S_{j}=0
\end{aligned}
$$

where $f_{S, A}$ and $f_{S, B}$ are load case depending factors.

Division by $R$ and multiplication by $E_{\mathrm{i}}$ after introducing of the quantity $\phi=E_{\mathrm{i}} / E_{\mathrm{m}}$ and performing a couple of algebraic re-arrangements the equation system is re-written as

$$
\begin{aligned}
& -\left[1-\phi\left(1-C_{p}\right)\right] \sigma_{z}^{*}+\left[2 v_{\mathrm{i}}-2 \phi\left(v_{\mathrm{m}}+C_{q}\right)\right] \sigma_{r}^{*}+\phi f_{S, A} S_{j}=0 \\
& \quad\left[v_{\mathrm{i}}-\phi\left(v_{\mathrm{m}}+C_{q}\right)\right] \sigma_{z}^{*}-\left[1-v_{\mathrm{i}}-\phi\left(1-v_{\mathrm{m}}-C_{p}-C_{q}\right)\right] \sigma_{r}^{*}+\phi f_{S, B} S_{j}=0
\end{aligned}
$$

Introducing the expressions

$$
\begin{aligned}
& \psi_{1}=-1-\phi\left[\frac{13-2 v_{\mathrm{m}}-15 v_{\mathrm{m}}^{2}}{2\left(7-5 v_{\mathrm{m}}\right)}\right]=-1+\phi\left(1-C_{p}\right) \\
& \psi_{2}=v_{\mathrm{i}}-\phi\left[\frac{3-2 v_{\mathrm{m}}-5 v_{\mathrm{m}}^{2}}{2\left(7-5 v_{\mathrm{m}}\right)}\right]=v_{\mathrm{i}}-\phi \cdot\left(v_{\mathrm{m}}+C_{q}\right) \\
& \psi_{3}=-1+v_{\mathrm{i}}-\phi\left[\frac{5-5 v_{\mathrm{m}}^{2}}{7-5 v_{\mathrm{m}}}\right]=-1+v_{\mathrm{i}}+\phi \cdot\left(1-v_{\mathrm{m}}-C_{p}-C_{q}\right)
\end{aligned}
$$


reduces the equation system to

$$
\begin{aligned}
& \psi_{1} \sigma_{z}^{*}+2 \psi_{2} \sigma_{r}^{*}+\phi f_{S, A} S_{j}=0 \\
& \psi_{2} \sigma_{z}^{*}+\psi_{3} \sigma_{r}^{*}+\phi f_{S, B} S_{j}=0
\end{aligned}
$$

5.1 Solution of the axial loading case $S_{z}$

The load factors of this load case are

$$
\begin{aligned}
& f_{S, A}=C_{p}=\frac{3}{2}\left(1-v_{\mathrm{m}}\right)\left[\frac{9+5 v_{\mathrm{m}}}{7-5 v_{\mathrm{m}}}\right] \\
& f_{S, B}=C_{q}=-\frac{3}{2}\left(1-v_{\mathrm{m}}\right)\left[\frac{1+5 v_{\mathrm{m}}}{7-5 v_{\mathrm{m}}}\right]
\end{aligned}
$$

After completion the equation system reads

(A)

$$
\begin{aligned}
& \psi_{1} \sigma_{z}^{*}+2 \psi_{2} \sigma_{r}^{*}+\phi C_{p} S_{z}=0 \\
& \psi_{2} \sigma_{z}^{*}+\psi_{3} \sigma_{r}^{*}+\phi C_{q} S_{z}=0
\end{aligned}
$$

The solution of the equations yields

$$
\begin{aligned}
\sigma_{z}^{*} & =\frac{\phi\left[2 \psi_{2} C_{q}-\psi_{3} C_{p}\right]}{\psi_{1} \psi_{3}-2 \psi_{2}^{2}} S_{z} \\
\sigma_{r}^{*} & =\frac{\phi\left[\psi_{2} C_{p}-\psi_{1} C_{q}\right]}{\psi_{1} \psi_{3}-2 \psi_{2}^{2}} S_{z}
\end{aligned}
$$

or expressed in a handbook-compatible formulation

$$
\begin{aligned}
& \sigma_{z}^{*}=K_{z}\left(S_{z}\right) \cdot S_{z}=K_{z}^{z} \cdot S_{z} \\
& \sigma_{r}^{*}=K_{r}\left(S_{z}\right) \cdot S_{z}=K_{r}^{z} \cdot S_{z}
\end{aligned}
$$

with

$$
\begin{aligned}
& K_{z}^{z}=\frac{\phi\left[2 \psi_{2} C_{q}-\psi_{3} C_{p}\right]}{\psi_{1} \psi_{3}-2 \psi_{2}^{2}} \\
& K_{r}^{z}=\frac{\phi\left[\psi_{2} C_{p}-\psi_{1} C_{q}\right]}{\psi_{1} \psi_{3}-2 \psi_{2}^{2}}
\end{aligned}
$$

Plots of $K_{z}$ and $K_{r}$ are shown in the diagrams in Figs. 16 and 17. Besides the reference curve with $v_{\mathrm{i}}=v_{\mathrm{m}}=0.3$ two other curves with extreme Poisson's ratio combinations of $v_{\mathrm{i}}=0.5$ and $v_{\mathrm{m}}=0.1$ or $v_{\mathrm{i}}=0.1$ and $v_{\mathrm{m}}=0.5$ are given, showing the bandwidth of theoretical values. The solution was confirmed by a series of numerical calculations by the finite element method, the results of the latter are indicated with symbols in Figs. 16 and 17 . 


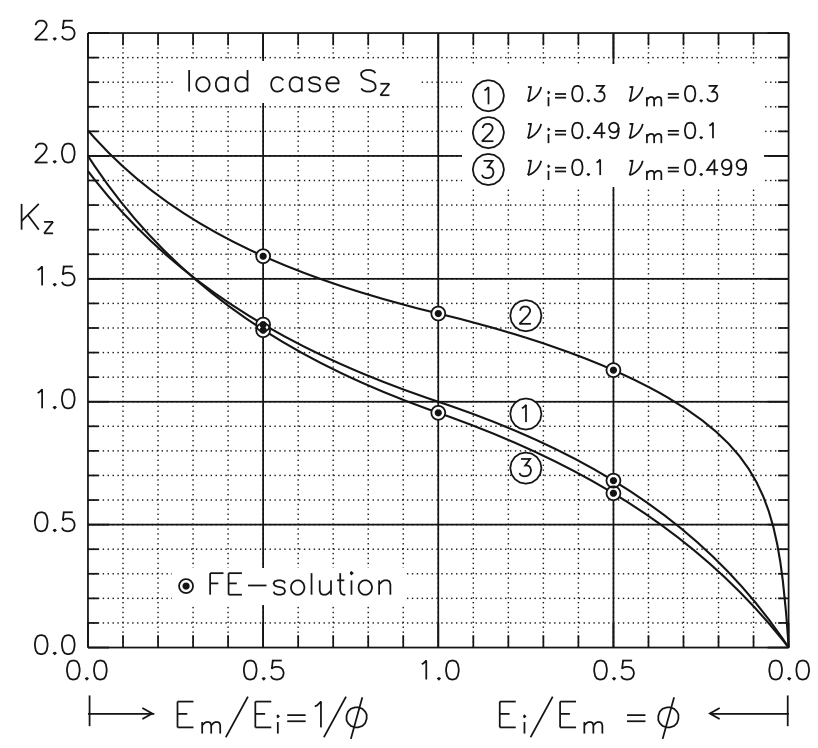

Fig. 16 Diagram of $K_{z}$-values for axial loading $S_{z}$

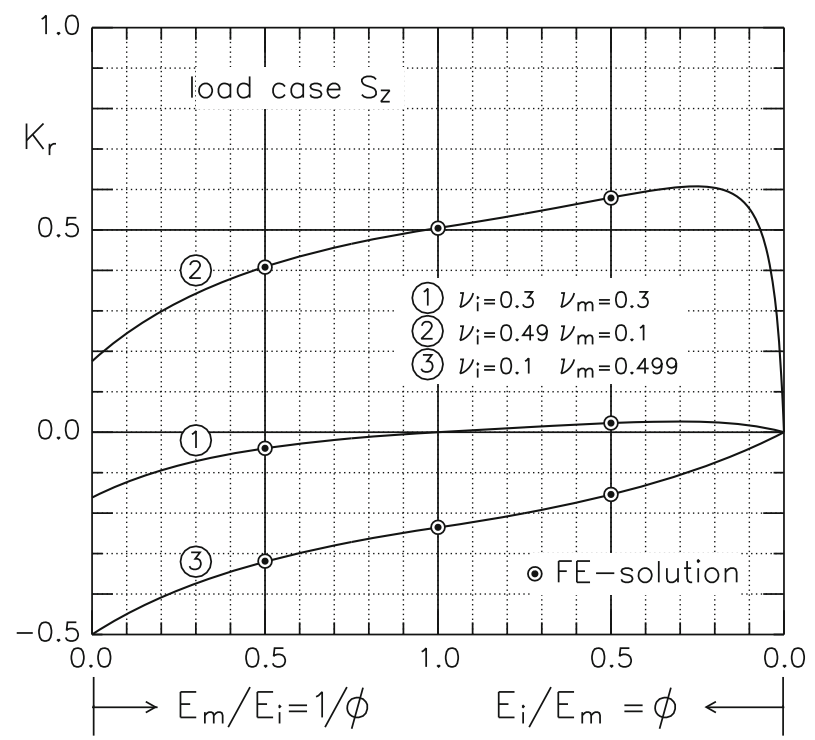

Fig. 17 Diagram of $K_{r}$-values for axial loading $S_{z}$

5.2 Solution of the axial-symmetrical radial loading case $S_{r}$

In this load case, the load factors become

$$
\begin{aligned}
& f_{S, A}=2 C_{q}=3\left(1-v_{\mathrm{m}}\right) \frac{9+5 v_{\mathrm{m}}}{7-5 v_{m}} \\
& f_{S, B}=C_{p}+C_{q}=3 \frac{1-v_{\mathrm{m}}}{7-5 v_{\mathrm{m}}}
\end{aligned}
$$

and after inserting in Eqs. (73) and (74), the equation system arises

$$
\psi_{1} \sigma_{z}^{*}+2 \psi_{2} \sigma_{r}^{*}+2 \phi C_{q} S_{r}=0
$$

(B)

$$
\psi_{2} \sigma_{z}^{*}+\psi_{3} \sigma_{r}^{*}+\phi\left(C_{p}+C_{q}\right) S_{r}=0
$$




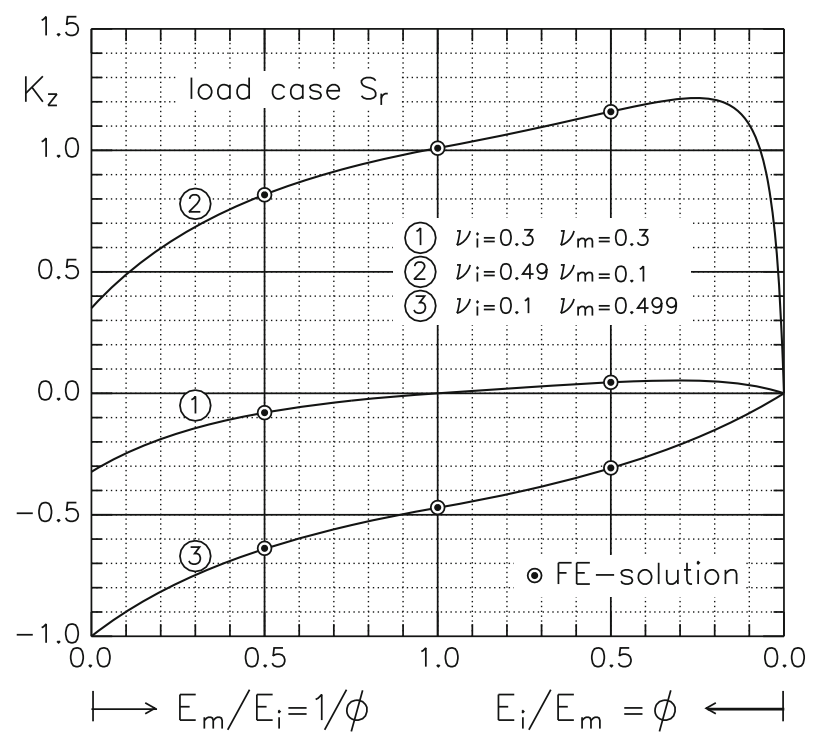

Fig. 18 Diagram of $K_{z}$-values for radial loading $S_{r}$

From these equations, the sought-after stress components $\sigma_{z}^{*}$ and $\sigma_{r}^{*}$ result in

$$
\begin{aligned}
\sigma_{z}^{*} & =\frac{\phi\left[2 \psi_{2}\left(C_{p}+C_{q}\right)-2 \psi_{3} C_{q}\right]}{\psi_{1} \psi_{3}-2 \psi_{2}^{2}} S_{r} \\
\sigma_{r}^{*} & =\frac{\phi\left[2 \psi_{2} C_{q}-\psi_{1}\left(C_{p}+C_{q}\right)\right]}{\psi_{1} \psi_{3}-2 \psi_{2}^{2}} S_{r}
\end{aligned}
$$

or formulated in $K$-values

$$
\begin{aligned}
& \sigma_{z}^{*}=K_{z}\left(S_{r}\right) \cdot S_{r}=K_{z}^{r} \cdot S_{r} \\
& \sigma_{r}^{*}=K_{r}\left(S_{r}\right) \cdot S_{r}=K_{r}^{r} \cdot S_{r}
\end{aligned}
$$

with

$$
\begin{gathered}
K_{z}^{r}=\frac{\phi\left[2 \psi_{2}\left(C_{p}+C_{q}\right)-2 \psi_{3} C_{q}\right]}{\psi_{1} \psi_{3}-2 \psi_{2}^{2}} \\
K_{r}^{r}=\frac{\phi\left[2 \psi_{2} C_{q}-\psi_{1}\left(C_{p}+C_{q}\right)\right]}{\psi_{1} \psi_{3}-2 \psi_{2}^{2}}
\end{gathered}
$$

Graphs of $K_{z}\left(S_{r}\right)$ and $K_{r}\left(S_{r}\right)$ are given in Figs. 18 and 19. Again curves of extreme Poisson's ratios show the variety of theoretically possible values in comparison with usual Poisson's ratios for steel as $v_{\mathrm{i}}=0.3$ and $v_{\mathrm{m}}=0.3$.

\section{Transformation of the stress state in the inhomogeneity to the stress state at the interface layer of the matrix}

As inhomogeneity and matrix have different elastic material parameters, the knowledge of the inhomogeneity stress state alone is not sufficient to asses the failure of the component. The highest stress concentrations in the matrix are located at the interface between inhomogeneity and matrix. To include the assessment of the matrix, it seems sufficient to transform the stresses of the inhomogeneity to the stresses at the matrix layer of the interface. 


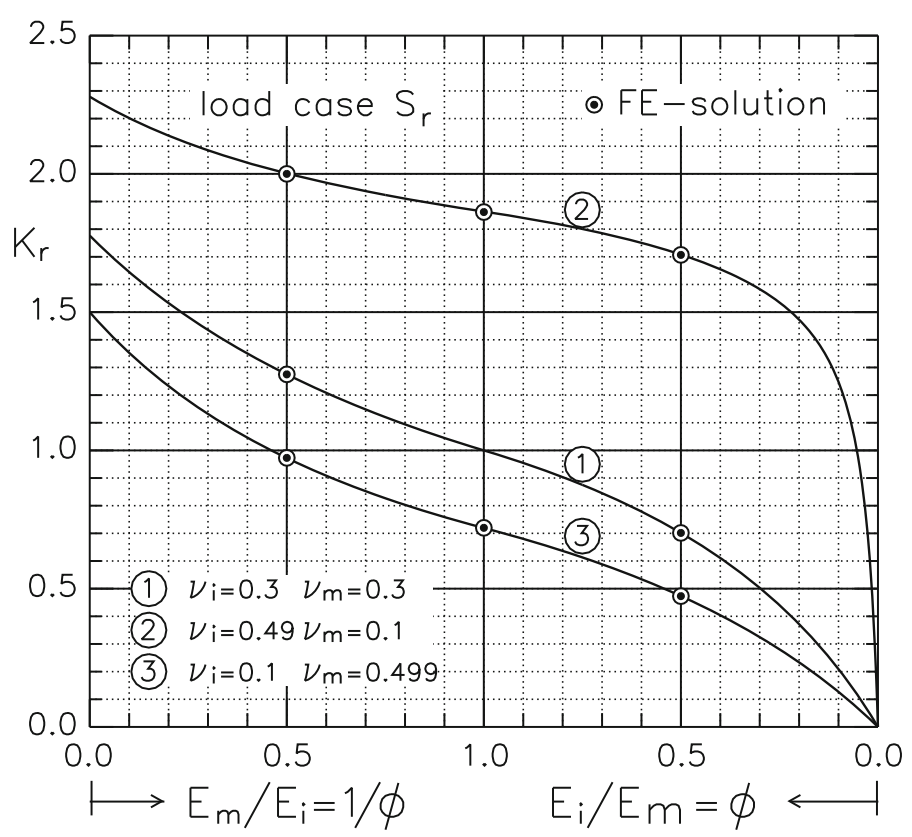

Fig. 19 Diagram of $K_{r}$-values for radial loading $S_{r}$

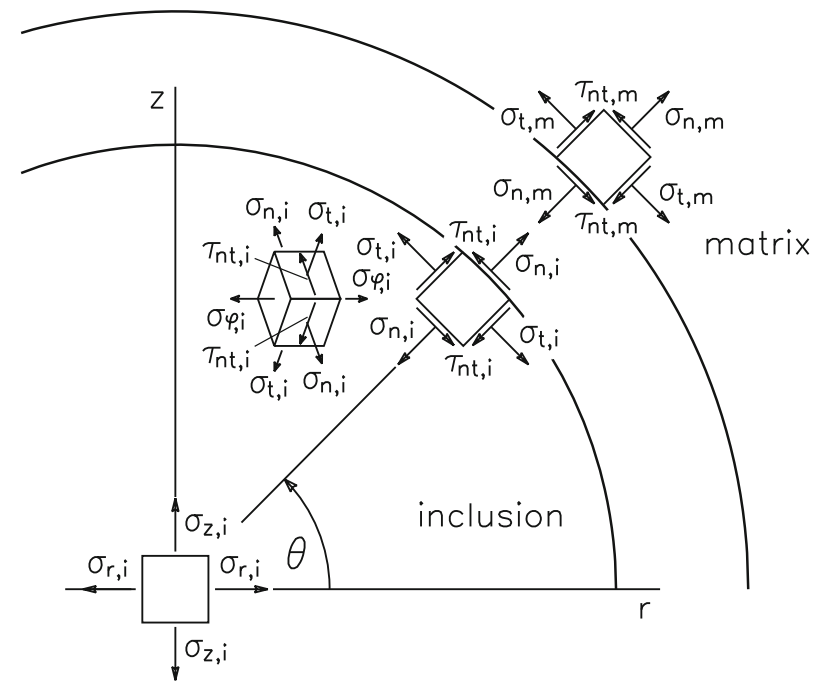

Fig. 20 Transformation of the inhomogeneity stress state to the stress components of the matrix at the interface layer

The stress state of the inhomogeneity can be transferred to the adjacent layer of the matrix, Fig. 20, by the transition conditions of the stress components in normal and tangential direction considering actio-reactio principle and compatibility of deformations. The normal and tangential direction of a given point of the surface $C$ is determined by a vertical angle $\theta$. Because of the axis-symmetrical system, the stress component in circumferential direction is

$$
\sigma_{\varphi, i}=\sigma_{r, i}=\text { const }
$$

inside the inhomogeneity. The inhomogeneity stresses $\sigma_{n, i}, \sigma_{t, i}$ and $\tau_{n t, i}$ are correlated with the components $\sigma_{z, i}$ and $\sigma_{r, i}$ by the known transformation relations

$$
\sigma_{n, i}=\frac{\sigma_{r, i}+\sigma_{z, i}}{2}+\frac{\sigma_{r, i}-\sigma_{z, i}}{2} \cos (2 \theta)
$$




$$
\begin{aligned}
\sigma_{t, i} & =\frac{\sigma_{r, i}+\sigma_{z, i}}{2}-\frac{\sigma_{r, i}-\sigma_{z, i}}{2} \cos (2 \theta) \\
\tau_{n t, i} & =-\frac{\sigma_{r, i}-\sigma_{z, i}}{2} \sin (2 \theta)
\end{aligned}
$$

or expressed by factors $K_{z}^{j}$ and $K_{r}^{j}$ of the relevant load case $j$

$$
\begin{aligned}
\sigma_{n, i} & =\left[\frac{K_{r}^{j}+K_{z}^{j}}{2}+\frac{K_{r}^{j}-K_{z}^{j}}{2} \cos (2 \theta)\right] S_{j} \\
\sigma_{t, i} & =\left[\frac{K_{r}^{j}+K_{z}^{j}}{2}-\frac{K_{r}^{j}-K_{z}^{j}}{2} \cos (2 \theta)\right] S_{j} \\
\tau_{n t, i} & =-\left[\frac{K_{r}^{j}-K_{z}^{j}}{2} \sin (2 \theta)\right] S_{j}
\end{aligned}
$$

A more direct calculation of the sum $K_{r}+K_{z}$ and difference $K_{r}-K_{z}$ can be chosen by special $K$-factors $K_{r+z}$ and $K_{r-z}$. In the axial loading case, $S_{z}$

$$
\begin{aligned}
& K_{r+z}\left(S_{z}\right)=\frac{K_{r}^{z}+K_{z}^{z}}{2}=\frac{\phi\left[\left(\psi_{2}-\psi_{3}\right) C_{p}-\left(\psi_{1}-2 \psi_{2}\right) C_{q}\right]}{2\left(\psi_{1} \psi_{3}-2 \psi_{2}^{2}\right)} \\
& K_{r-z}\left(S_{z}\right)=\frac{K_{r}^{z}-K_{z}^{z}}{2}=\frac{\phi\left[\left(\psi_{2}+\psi_{3} C_{p}-\left(\psi_{1}+2 \psi_{2}\right) C_{q}\right]\right.}{2\left(\psi_{1} \psi_{3}-2 \psi_{2}^{2}\right)}
\end{aligned}
$$

for the axialsymmetrical loading $S_{r}$ by

$$
\begin{aligned}
& K_{r+z}\left(S_{r}\right)=\frac{K_{r}^{r}+K_{z}^{r}}{2}=\frac{\phi\left[2\left(\psi_{2}-\psi_{3}\right) C_{q}-\left(\psi_{1}-2 \psi_{2}\right)\left(C_{p}+C_{q}\right)\right]}{2\left(\psi_{1} \psi_{3}-2 \psi_{2}^{2}\right)} \\
& K_{r-z}\left(S_{r}\right)=\frac{K_{r}^{r}-K_{z}^{r}}{2}=\frac{\phi\left[\left(2\left(\psi_{2}+\psi_{3}\right) C_{q}-\left(\psi_{1}+2 \psi_{2}\right)\left(C_{p}+C_{q}\right)\right]\right.}{2\left(\psi_{1} \psi_{3}-2 \psi_{2}^{2}\right)}
\end{aligned}
$$

Now, the stress components on the i surface inside the inhomogeneity become

$$
\begin{aligned}
\sigma_{n, i} & =\left[K_{r+z}\left(S_{j}\right)+K_{r-z}\left(S_{j}\right) \cos (2 \theta)\right] S_{j} \\
\sigma_{t, i} & =\left[K_{r+z}\left(S_{j}\right)-K_{r-z}\left(S_{j}\right) \cos (2 \theta)\right] S_{j} \\
\tau_{n t, i} & =-\left[K_{r-z}\left(S_{j}\right) \sin (2 \theta)\right] S_{j}
\end{aligned}
$$

To the stress components on the matrix side of the interface, actio-reactio principle implies

$$
\begin{aligned}
\sigma_{n, m, c} & =\sigma_{n, i} \\
\tau_{n t, m, c} & =\tau_{n t, i},
\end{aligned}
$$

while for both of the other stress components, it has to be noticed

$$
\begin{aligned}
\sigma_{t, m, c} & \neq \sigma_{t, i} \\
\sigma_{\varphi, m, c} & \neq \sigma_{\varphi, i}
\end{aligned}
$$

Compatible deformations postulate

$$
\begin{aligned}
\varepsilon_{t, m, c} & =\varepsilon_{t, i} \\
\varepsilon_{\varphi, m, c} & =\varepsilon_{\varphi, i}
\end{aligned}
$$

whereas the strain component in normal direction changes

$$
\varepsilon_{n, m, c} \neq \varepsilon_{n, i} .
$$




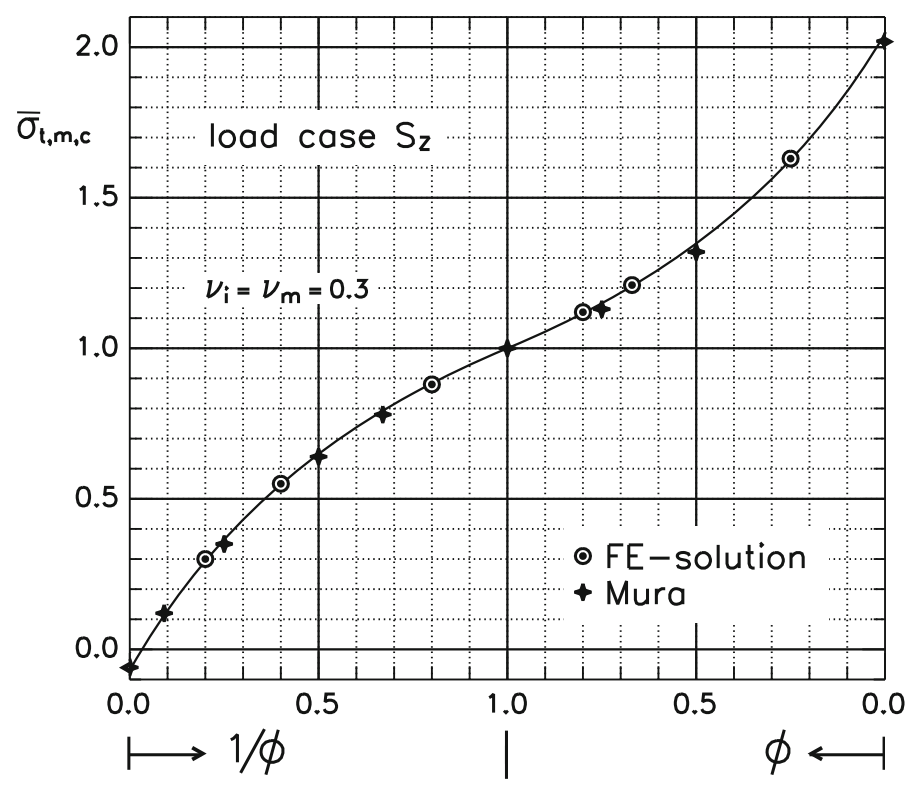

Fig. 21 Comparison of analytically calculated notch stresses $\sigma_{t, m, c}$ for $\theta=0$ with numerically obtained as well as literature [14] values, normalized by the applied load $S_{z}$

Using Eqs. (95)-(97), (99) and (100), the missing stress components can be determined by the stress-strain equations

$$
\begin{aligned}
\varepsilon_{t, i} & =\frac{1}{E_{\mathrm{i}}}\left[\sigma_{t, i}-v_{\mathrm{i}}\left(\sigma_{n, i}+\sigma_{\varphi, i}\right)\right] \\
\varepsilon_{t, m, c} & =\frac{1}{E_{\mathrm{m}}}\left[\sigma_{t, m, c}-v_{\mathrm{m}}\left(\sigma_{n, m, c}+\sigma_{\varphi, m, c}\right)\right] \\
\varepsilon_{\varphi, i} & =\frac{1}{E_{\mathrm{i}}}\left[\sigma_{\varphi, i}-v_{\mathrm{i}}\left(\sigma_{n, i}+\sigma_{t, i}\right)\right] \\
\varepsilon_{\varphi, m, c} & =\frac{1}{E_{\mathrm{m}}}\left[\sigma_{\varphi, m, c}-v_{\mathrm{m}}\left(\sigma_{n, m, c}+\sigma_{t i, m, c}\right)\right] .
\end{aligned}
$$

As $\varepsilon_{t, m, c}=\varepsilon_{t, i}$ and $\varepsilon_{\varphi, m, c}=\varepsilon_{\varphi, i}$, the relations can be used for the following equation system

$$
\begin{aligned}
& \sigma_{t, m, c}-v_{\mathrm{m}} \sigma_{\varphi, m, c}=\frac{E_{\mathrm{m}}}{E_{\mathrm{i}}}\left[\sigma_{t, i}-v_{\mathrm{i}}\left(\sigma_{n, i}+\sigma_{\varphi, i}\right)\right]+v_{\mathrm{m}} \sigma_{n, m, c} \\
& \sigma_{\varphi, m, c}-v_{\mathrm{m}} \sigma_{t, m, c}=\frac{E_{\mathrm{m}}}{E_{\mathrm{i}}}\left[\sigma_{\varphi, i}-v_{\mathrm{i}}\left(\sigma_{n, i}+\sigma_{t, i}\right)\right]+v_{\mathrm{m}} \sigma_{n, m, c} .
\end{aligned}
$$

The solution leads to

$$
\begin{aligned}
\sigma_{t, m, c} & =\frac{1}{1-v_{\mathrm{m}}^{2}}\left[\frac{E_{\mathrm{m}}}{E_{\mathrm{i}}}\left[\sigma_{t, i}-v_{\mathrm{i}}\left(\sigma_{\varphi, i}+\sigma_{n, i}\right)\right]+v_{\mathrm{m}} \frac{E_{\mathrm{m}}}{E_{\mathrm{i}}}\left[\sigma_{\varphi, i}-v_{\mathrm{i}}\left(\sigma_{t, i}+\sigma_{n, i}\right)\right]+v_{\mathrm{m}}\left(1+v_{\mathrm{m}}\right) \sigma_{n, m, c}\right] \\
\sigma_{\varphi, m, c} & =\frac{1}{1-v_{\mathrm{m}}^{2}}\left[\frac{E_{\mathrm{m}}}{E_{\mathrm{i}}}\left[\sigma_{\varphi, i}-v_{\mathrm{i}}\left(\sigma_{t, i}+\sigma_{n, i}\right)\right]+v_{\mathrm{m}} \frac{E_{\mathrm{m}}}{E_{\mathrm{i}}}\left[\sigma_{t, i}-v_{\mathrm{i}}\left(\sigma_{\varphi, i}+\sigma_{n, i}\right)\right]+v_{\mathrm{m}}\left(1+v_{\mathrm{m}}\right) \sigma_{n, m, c}\right]
\end{aligned}
$$

The stress component $\sigma_{t, m, c}$ of Eq. (122), normalized by the applied load $S_{z}$, allows for $\theta=0$ a comparison with Mura's results given in curve plots, page 191 in [14]. In Fig. 21, the course of Eq. (122) in dependency of $\phi=E_{\mathrm{i}} / E_{\mathrm{m}}$ is compared with the results of Mura as well as with some values from finite element analyses. The results described by Eq. (122) are identical to Mura's results within the accuracy of his graphics. This is not too surprising as both approaches deliver exact solutions. 


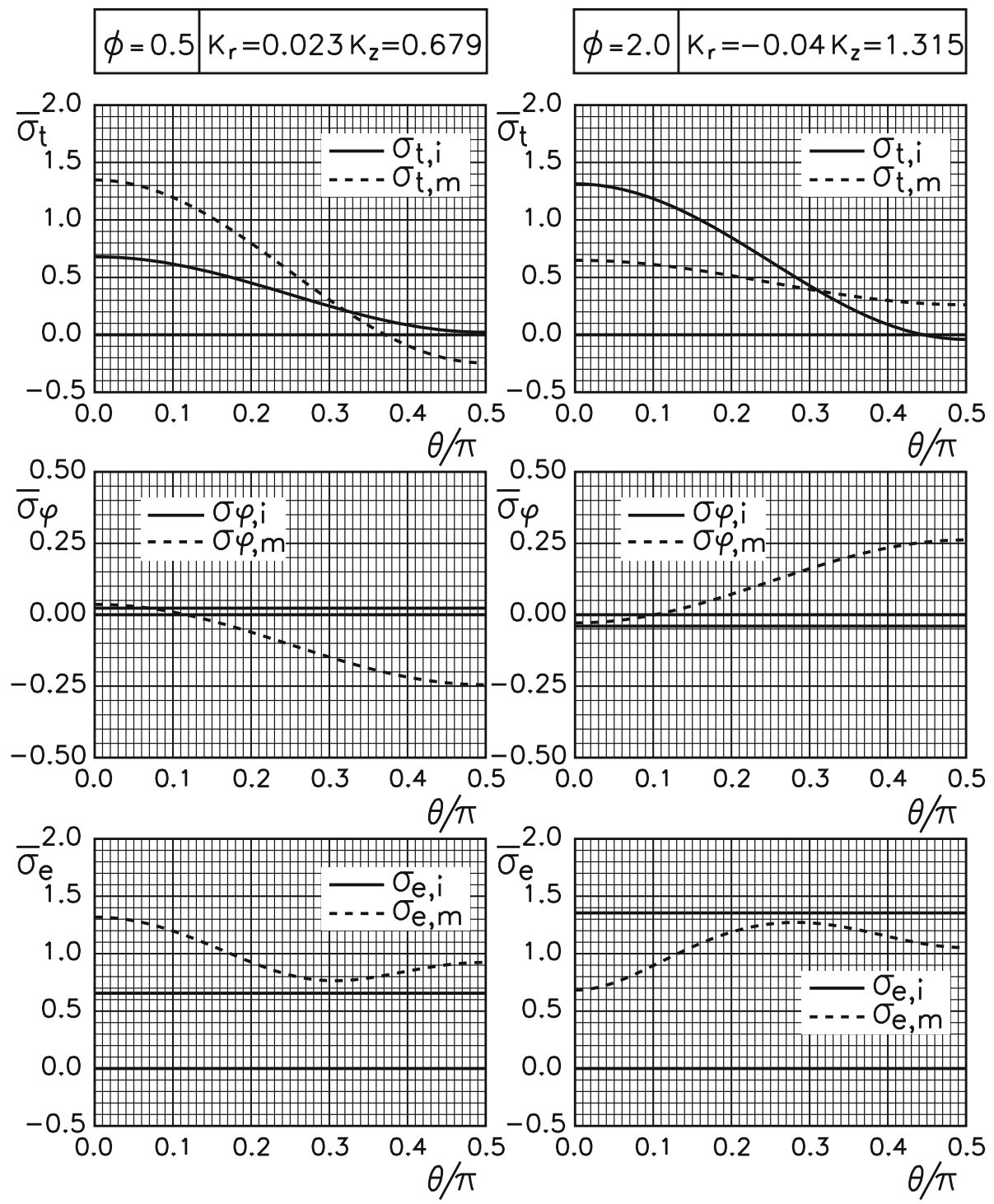

Fig. 22 Stress functions $\bar{\sigma}_{t}, \bar{\sigma}_{\varphi}$ in tangential and perimeter direction and according to the maximum distortion strain energy criterion $\bar{\sigma}_{e}$ for axial loading $S_{z}$

A material-specific failure theory will require to derive an appropriate equivalent stress. For example, the maximum distortion strain energy criterion is described by the equivalent stress $\sigma_{e}$ according to von Mises

$$
\sigma_{e}=\sqrt{\frac{1}{2}\left[\left(\sigma_{n}-\sigma_{t}\right)^{2}+\left(\sigma_{t}-\sigma_{\varphi}\right)^{2}+\left(\sigma_{\varphi}-\sigma_{n}\right)^{2}+6 \tau_{n t}^{2}\right]}=\sqrt{3 \cdot J_{2}^{\prime}}
$$

where $J_{2}^{\prime}$ is the second invariant of the stress deviator.

The calculated stress components are plotted in the diagrams of Fig. 22 for axial loading by $S_{z}$ and of Fig. 23 for axis-symmetrical radial loading $S_{r}$ both in their dependence of the vertical angle $\theta$. The left-side diagrams apply to soft inhomogeneities with $\phi=E_{\mathrm{i}} / E_{\mathrm{m}}=0.5$, the right-side ones to hard inhomogeneities with $\phi=E_{\mathrm{i}} / E_{\mathrm{m}}=2.0$. In each chart the graphs of a stress component of the inhomogeneities (solid line) is confronted that at the interface of the matrix (dashed line) demonstrating the deviations as a result of the differing Youngs moduli of inhomogeneities and matrix.

The equivalent stress $\sigma_{e}$ shown in the bottom diagrams might be chosen for an evaluation of the material's strength capacity. Soft inhomogeneities with $\phi=E_{\mathrm{i}} / E_{\mathrm{m}}<1$ move the stress peaks of $\sigma_{e}$ to the matrix side, 


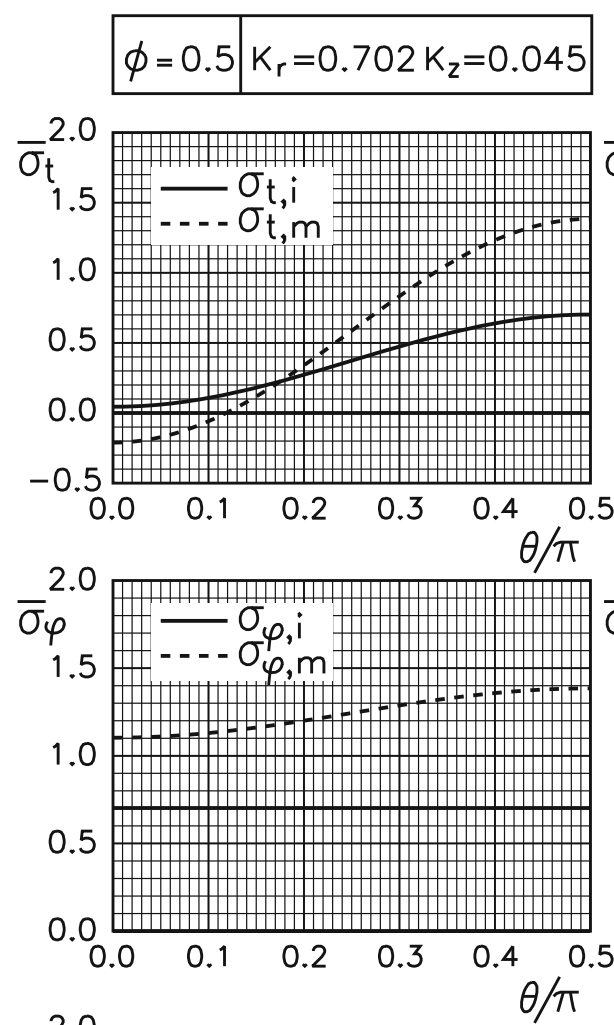

$$
\begin{array}{|l|l|}
\hline \phi=2.0 & K_{r}=1.275 K_{z}=-0.08 \\
\hline
\end{array}
$$
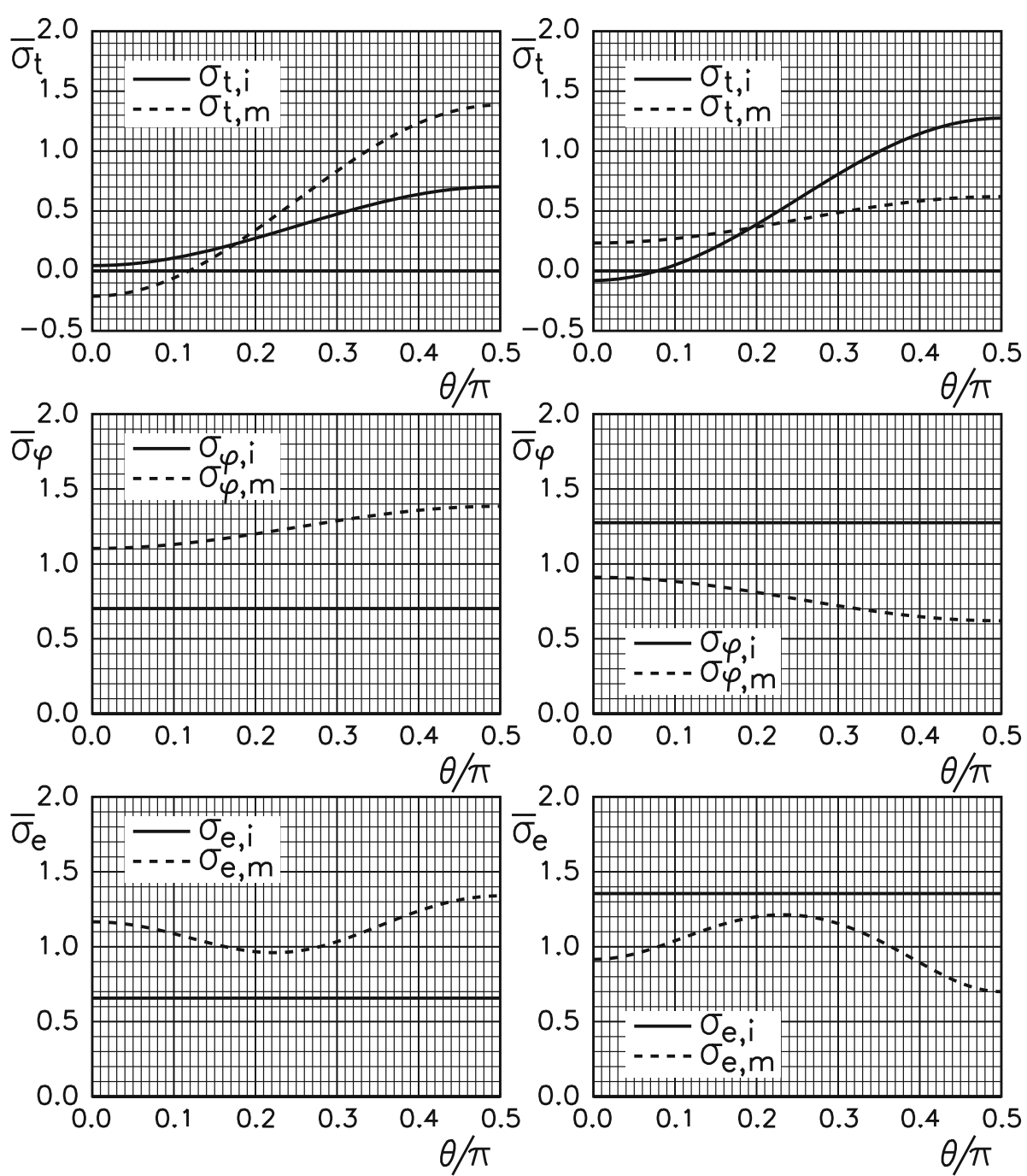

Fig. 23 Stress functions $\bar{\sigma}_{t}, \bar{\sigma}_{\varphi}$ in tangential and perimeter direction and according to the maximum distortion strain energy criterion $\bar{\sigma}_{e}$ for radial loading $S_{r}$

while hard inhomogeneities $\phi=E_{\mathrm{i}} / E_{\mathrm{m}}>1$ have an attractive effect with higher stress levels relieving the matrix. But absolute statements require the knowledge of and the comparison with the strength limits of both material constituents and the interface itself.

It is remarkable that the equivalent stress of the inhomogeneities in Fig. 22 for the load case $S_{z}$ is identical to that of Fig. 23 for the load case $S_{r}$. The background of this equivalence is that the stress state caused by a single load $S_{x}$ (generating an equivalent stress as by $S_{z}$ ) remains unchanged by a superposition of a second load $S_{y}$, whereas the stress components change.

Non-local failure hypotheses not only require information for stress or strain values at the critical location, e.g., at the interface for matrix failure, on which the focus has been placed so far. Only by applying such nonlocal hypotheses can, for example, the fatigue strength of materials and components be described realistically, see e.g., $[15,16]$. As mentioned in Sect. 4.1, all field variables are available in analytical form, provided the input, $\sigma_{z}^{*}$ and $\sigma_{r}^{*}$, is known. Only a more or less virtuoso superposition of NEUBER's solution equations [12] for the spherical cavities becomes necessary. Figure 24 shows the curves for the stress component $\sigma_{z}(r)$, for $\theta=0$, normalized by the applied load $S_{z}$, for various $\Phi$-values. The dot-in-circle markers $\odot$ indicate that FE calculations have been performed. The graphs of the results of the converged numerical solution cannot 


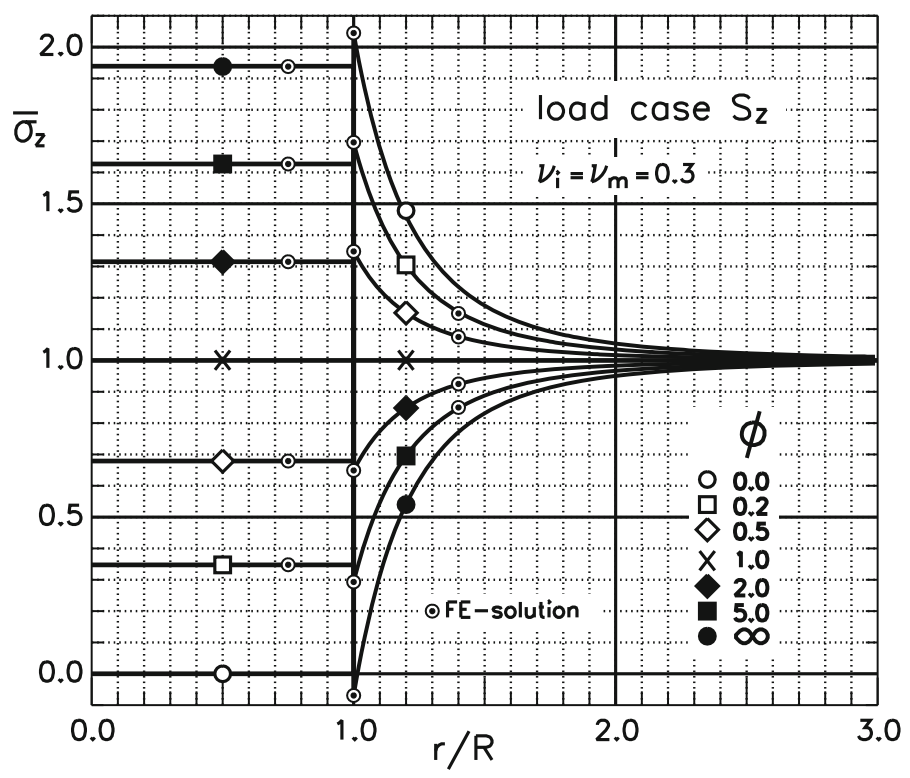

Fig. 24 Curves of normalized stress component $\bar{\sigma}_{z}(r)$, for $\theta=0$, for various $\Phi$-values under axial loading $S_{z}$

be distinguished from the graph of the analytical solution, independent of any reasonable resolution of the graphics.

\section{Conclusions}

Up to now, the known solutions for a spherical inhomogeneity in an infinite solid have found little attention in practical failure assessments because of their disregard in the handbooks for engineering design. This lack may be attributed to the complicated mathematical methods of derivation and the incommodious depiction of the solutions. The exact solution can, however, also be presented in a more engineering oriented way of analysis considering the special deformation characteristics of the spherical inhomogeneity problem. The method used is based only on the special solution for a spherical cave from NEUBER's solution [12] for the axial-symmetrical deep ellipsoidal inner notch published 1937 . Theoretically at that time, the precondition to solve the corresponding inhomogeneity problem was given.

As soon as the analytical solution for a remote homogenous loading with $S_{z}$ is known, the solutions for $S_{x^{-}}$ or $S_{y}$-loading can be obtained by simple permutation of indexes. The three solutions can further be superposed to provide the solution for an arbitrary remote homogeneous principal stress loading case. Any given remote homogeneous loading situation can be transformed to a principal stress loading case. After solving the latter, back-transformation of the field variables into the coordinate system of the given stress components provides the solution for this case, too. In this sense, the presented solution is comprehensive.

The present result is a compact formula including three simple influence factors depending on Young's moduli and Poisson's ratios which describes the stress state in the inhomogeneity in form of stress concentration factors. The stress state at the interface of the matrix can also be expressed in terms of concentration factors. It is expected that the clearly structured derivation supports the publication in handbooks and the application in practice.

Funding Open Access funding enabled and organized by Projekt DEAL.

Open Access This article is licensed under a Creative Commons Attribution 4.0 International License, which permits use, sharing, adaptation, distribution and reproduction in any medium or format, as long as you give appropriate credit to the original author(s) and the source, provide a link to the Creative Commons licence, and indicate if changes were made. The images or other third party material in this article are included in the article's Creative Commons licence, unless indicated otherwise in a credit line to the material. If material is not included in the article's Creative Commons licence and your intended use is not permitted 
by statutory regulation or exceeds the permitted use, you will need to obtain permission directly from the copyright holder. To view a copy of this licence, visit http://creativecommons.org/licenses/by/4.0/.

\section{Compliance with ethical standards}

Conflict of interest On behalf of all authors, the corresponding author states that there is no conflict of interest.

\section{References}

1. Goodier, J.N.: Concentration of stress around spherical and cylindrical inclusions and flaws. J. Appl. Mech. Trans. ASME 55, A-39 (1933)

2. Edwards, R.H.: Stress concentration around spheroidal inclutions and cavities. J. Appl. Mech. Trans. ASME 18, 19 (1951)

3. Sadowsky, M.A., Sternberg, E.: Stress concentration around an elllipsoidal cavity in an infinite body under arbitrary plane stress perpendicular to the axis of the cavity. J. Appl. Mech. Trans. ASME 69, A-191 (1947)

4. Eshelby, J.D.: The determination of the elastic field of an ellipsoidal inclusions and related problems. Proc. R. Soc. Ser. A 241, 376-396 (1957)

5. Amstutz, H.: Elastizitätstheoretische Lösung des kreisförmigen Einschlusses in unendlicher Scheibe mittels der Beziehungen zwischen Loch- und Einschlussproblem. Mater. Sci. Eng. Technol. 44(11), 903-913 (2013)

6. Amstutz, H., Vormwald, M.: Analysis of an elastic elliptical inclusion in an infinite elastic plate under uniform remote tension based on the solution of the corresponding cavity problem. J. Strain Anal. Eng. Des. 52(8), 515-527 (2017)

7. Budynas, R.G., Sadegh, A.M.: Roark's Formulas for Stress and Strain, 9th edn. McGraw Hill, New York (2020)

8. Pilkey, W.D., Pilkey, D.F.: Peterson's Stress Concentration Factors, 3rd edn. Wiley, Hoboken (2008)

9. Murakami, Y.: Theory of Elasticity and Stress Concentration. Wiley, Hoboken (2016)

10. Kachanov, M., Shafiro, B., Tsukrov, I.: Handbook of Elasticity Solutions. Springer, Berlin (2003)

11. Boresi, A.P., Schmidt, R.J.: Advanced Mechanics of Materials. Wiley, Hoboken (2002)

12. Neuber, H.: Kerbspannungslehre. Springer, Berlin (1937)

13. Ma, L.F., Korsunsky, A.M.: The principle of equivalent eigenstrain for inhomogeneous inclusion problems. Int. J. Solids Struct. 51, 4477-4484 (2014)

14. Mura, T.: Micromechanics of Defects in Solids. Kluwer, Dordrecht (1987)

15. Taylor, D.: The Theory of Critical Distances. Elsevier, Amsterdam (2007)

16. Hertel, O., Vormwald, M.: Statistical and geometrical size effects in notched members based on weakest-link and short-crack modelling. Eng. Fract. Mech. 95, 72-83 (2012)

Publisher's Note Springer Nature remains neutral with regard to jurisdictional claims in published maps and institutional affiliations. 